\title{
49. RELATIONSHIP BETWEEN THE VARIATION OF UNDRAINED SHEAR STRENGTH, ORGANIC CARBON CONTENT, AND THE ORIGIN AND FREQUENCY OF ENIGMATIC NORMAL FAULTS IN FINE-GRAINED SEDIMENTS FROM ADVANCED PISTON CORES FROM THE EASTERN MEDITERRANEAN ${ }^{1}$
}

\author{
Achim Kopf, ${ }^{2}$ M. Ben Clennell, ${ }^{3}$ and Rachel Flecker ${ }^{4}$
}

\begin{abstract}
Layered successions of hemipelagic sediments from different sites drilled during Ocean Drilling Program Leg 160 investigating the Eastern Mediterranean, are intersected by numerous high-angle normal faults. The formation and frequency distribution of these faults is a function of lithologic variation and thus different sediment physical properties, such as their strength and brittleness. Sediment failure is generally observed where organic-rich layers (sapropels or sapropelic beds of $>2 \%$ organic carbon) are interbedded with the predominant nannofossil ooze. Undrained shear strength measurements (vane, pocket penetrometer) on split deep sea cores show that sapropels are significantly firmer than the surrounding oozes and clays (up to $40 \mathrm{kPa}$ stronger within the same depth interval). Values $>220 \mathrm{kPa}$ were obtained for the most resistant sapropel beds. Drained shear box tests conducted at $1 \mathrm{MPa}$ normal stress on remolded sediment, however, revealed lower peak and residual shear strength for the sapropel (253 and $<226 \mathrm{kPa}$, respectively) compared with nannofossil ooze (621 and >580 kPa, respectively). The internal angle of friction of the sapropel is comparable to that of the weakest clay minerals $\left(\phi^{\prime} \mathrm{p} 11 \pm 1^{\circ} ; \phi^{\prime} \mathrm{r} 10 \pm 1^{\circ}\right)$ whereas friction angles for the nannofossil ooze are higher $\left(\phi^{\prime} \mathrm{p} 28 \pm 1^{\circ}, \phi^{\prime} \mathrm{r} 30 \pm 1^{\circ}\right)$ and controlled by its high carbonate content.

The influence of organic matter on sediment shear strength is the subject of an ongoing debate. The structural observations and shear strength determinations on sediments recovered from the Eastern Mediterranean favor a positive correlation between the abundance of organic carbon and sediment strength. The effect of particle bonding is lessened when the original sedimentary fabric is destroyed (e.g., remolding before shear box test). Faulting takes place adjacent to sapropels and fault occurrence in the stratigraphic succession can be positively correlated with abundance of sapropels.
\end{abstract}

\section{INTRODUCTION}

Structural data collected from many ODP legs indicate that normal faults are common in unlithified marine sediments (Kopf and Flecker, 1996). Cores collected during the ODP Leg 160 investigation of the Eastern Mediterranean (Emeis, Robertson, Richter, et al., 1996) contain layered successions of hemipelagic sediments from different drill sites are intersected by abundant high-angle normal faults. Visual inspection of the cut core suggested that there was a spacial relationship between lithologic variation (i.e., contrasts in the physical properties of the sediments) and fault occurrence.

The relationship between shear strength of marine soft sediments and their organic content has been studied by several authors (Busch and Keller, 1981; Reimers, 1982; Rashid and Brown, 1975), however, both the results and their interpretations have proved variable. Busch and Keller $(1981 ; 1982)$ demonstrated that samples taken from the Peru margin had lower shear strength when $\mathrm{C}_{\text {org }}$ contents exceeded $2 \%$ (i.e., an inverse relationship between strength and organiccarbon content). In contrast, decomposition and increasing humification of organic components were proposed as possible explanations for the apparent overconsolidation and high shear strength observed in these same sediments by Reimers (1982) and Lee et al. (1990). In addition to the results for marine sediments, other investigators have demonstrated that increasing organic matter causes higher shear

${ }^{1}$ Robertson, A.H.F., Emeis, K.-C., Richter, C., and Camerlenghi, A. (Eds.), 1998. Proc. ODP, Sci. Results, 160: College Station, TX (Ocean Drilling Program).

${ }^{2}$ Geologisches Institut, Albert-Ludwigs-Universität Freiburg, Albertstrasse 23B, 79104 Freiburg, Federal Republic of Germany. (Present address: GEOMAR, Wischhofstrasse 1-3, 24148 Kiel, Federal Republic of Germany.) akopf@geomar.de dom

${ }^{3}$ Department of Earth Sciences, University of Leeds, Leeds LS2 9JT, United King-

${ }^{4}$ CASP, Department of Earth Sciences, University of Cambridge, Downing Street, Cambridge CB2 9EU, United Kingdom. strength in artificially prepared specimens (i.e., a positive relationship; Pusch, 1973; Rashid and Brown, 1975; Andersland et al., 1981).

Drained and undrained sediment shear strength measurements are important quantifiable geological parameters that affect the response of sediments to gravitational and tectonic stresses (e.g., Atkinson 1993; Maltman, 1994) and equally affect the coring technique used for their recovery (Kopf and Flecker, 1996). In this study, closely spaced measurements of undrained shear strength, structural observations on split cores, and results from organic geochemistry carried out during Leg 160 (Emeis, Robertson, Richter, et al., 1996) are related to peak and residual shear strength results from drained geotechnical shear box tests, and to previous laboratory tests on artificial mixtures and marine sediment samples (Busch and Keller, 1981; Andersland et al., 1981; Lee et al., 1990; Lee et al., 1993). A comparison between similar successions recovered by multiple coring at the same drill site, sheds light on systematic shear failure of sediment with particular physical properties and organic-carbon content.

\section{GEOLOGICAL SETTING}

During Leg 160, layered sediments were recovered from the uppermost part of the Pliocene-Quaternary succession at different locations in the Eastern Mediterranean (Fig. 1; Emeis, Robertson, Richter, et al., 1996). At Site 964, located on the Calabrian Ridge, four holes were drilled into the Pisano Plateau. A transect of four sites was drilled across the Eratosthenes Seamount in order to investigate the plateau area (Site 966), the upper northern slope (Site 965), a small ridge at the slope break (Site 967), and the Cyprus slope to the north of the seamount (Site 968; Fig. 1).

At each site, the upper part of the Pliocene-Quaternary succession consisted of interbedded hemipelagic nannofossil ooze and clay with sapropels (2-27 cm thick, with an approximate meter-scale spacing)

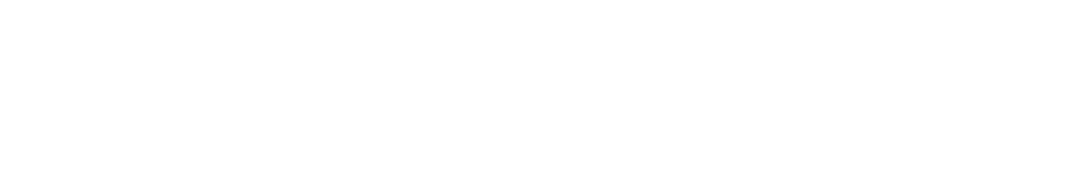




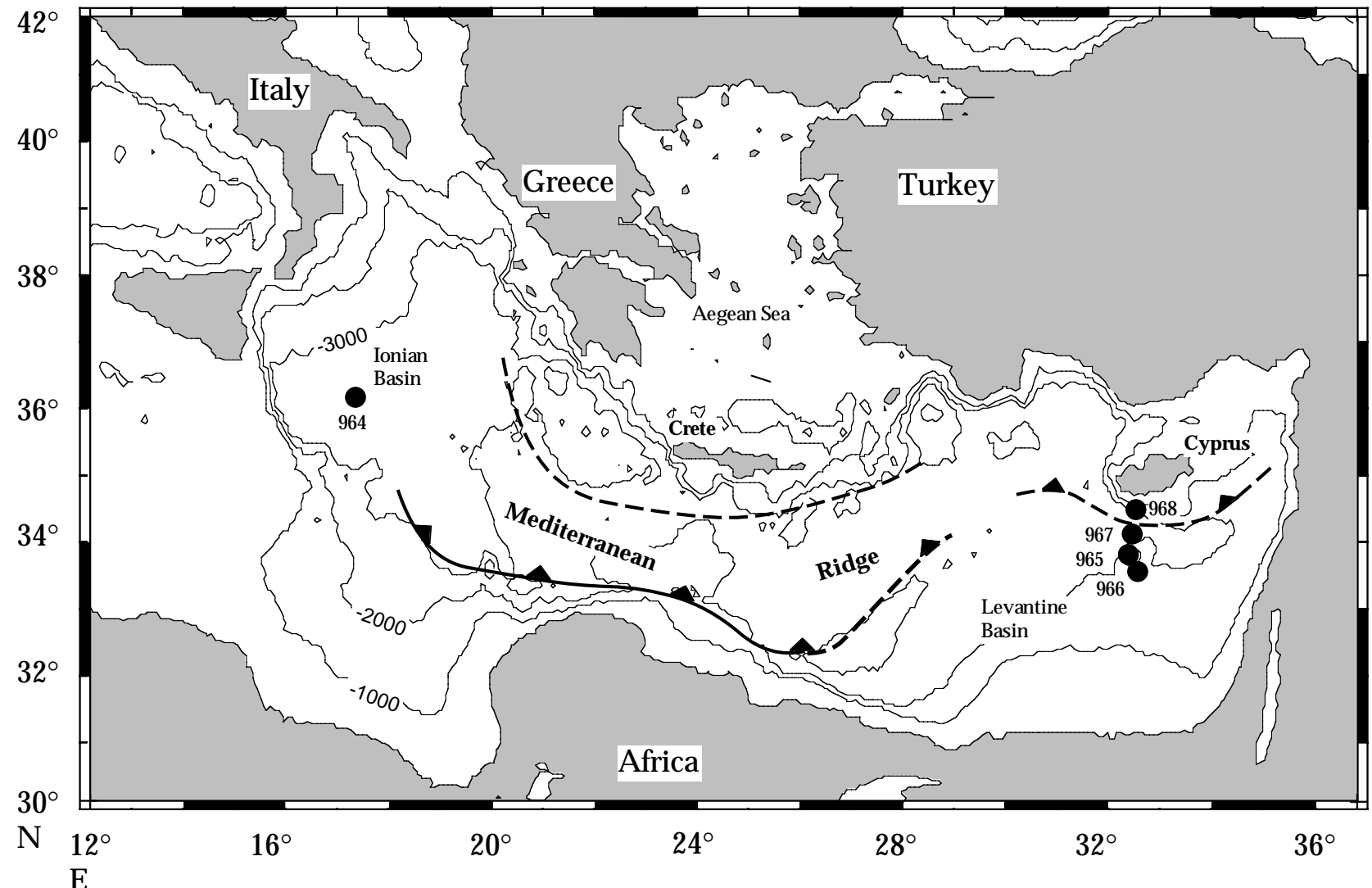

Figure 1. Location of Sites 964-968 drilled in the Eastern Mediterranean during Leg 160. Thin contour lines are in meters below sea level; bold lines describe the position of the trenches in the Eastern Mediterranean.

and occasional turbidites (e.g., Fig. 2). Normal faults appear to have formed mainly in intervals where sapropels are common. For this study of the relationship between fault formation and sediment physical properties, therefore, successions of interbedded nannofossil clays and sapropels that had been recovered by multiple coring were chosen for measurement. Nineteen cores (four cores from Site 964, two from Site 965, two from Site 966, five from Site 967, and six from Site968) were selected (see also Table 1). The depth range of the selected Pliocene-Quaternary sediments varied between 30 and 130 mbsf (Table 1).

\section{THEORETICAL BACKGROUND}

\section{Fault Formation and Relation to Strength Measurement}

Failure by shear occurs when stresses on potential planes of slip exceed the total strength that can be mobilized to resist movement (Hubbert, 1951; Jaeger and Cook, 1969; Maltman, 1994). Within the sediment pile, failure is anticipated where shear strengths are lowest. The total strength has components of friction and cohesion (the Mohr-Coulomb criterion). In fine-grained sediments, cohesion and friction are controlled by mineralogy and organic content (both particle shape and surface activity). Additionally, in all granular materials, there will be an element of mechanical work associated with shear-induced volume change (dilation) that affects the measured strength and depends upon the packing and structure of particles.

Mineral packing and particle size directly affect the strain-rate response of the sediment through the relationship between permeability and the drainage rate. If a material is permeable, or deformed at a slow rate such that pore fluid pressures induced by volumetric rear- rangements are dissipated, then the drained strength is mobilized. If a faster strain rate leads to sufficiently rapid changes such that the pore fluid cannot be fully dissipated, or the material is of low permeability or sealed in some way, then the deformation is considered undrained, and the resilience of the pore fluid must be taken into account (Yassir, 1990).

Loss of cohesion and thus lack of ductility lead to brittle behavior. Brittleness is contingent upon intrinsic material properties (e.g., clays are typically more ductile than silts, oozes and sands), stress history (i.e., the stress path to failure; Law, 1981), and strain rate. The stress history affects the strength and deformation path of fine-grained sediments in a coherent way, which can be predicted by the tenets of critical-state soil mechanics (Atkinson, 1993; Wood, 1990; Jones and Addis, 1986). In natural sediments these rules can be applied, provided that the effects of interparticle bonding, or structure (which will reduce the compressibility of the original sediment), increase the cohesive strength, and usually increase the brittleness are also taken into account (Burland, 1990; Lereouil and Vaughan, 1990).

From the above considerations we can make two important points. Firstly, the method of measurement chosen (vane shear, triaxial test, direct shear box) will markedly influence the measured strength of a sediment as the strain rate, stress path, and drainage state are unlikely to be exactly comparable. Second, natural (tectonic, gravitational) processes and human activities (coring, geotechnical tests) will impose deformation histories on a sediment, which can be interpreted in terms of the stress and strain-rate paths and the drainage condition. Different strength measurements will be pertinent to the interpretation of the different processes. For example, a sediment is assumed to be undergoing undrained shear when the advanced hydraulic piston coring (APC) device is used, as it penetrates the sedi- 
ment too quickly for fluid dispersal (Kopf, 1997). Undrained tests of strength are, therefore, valuable to interpret the structures generated by APC coring.

\section{METHODS}

\section{Fault Measurements}

Faults were measured following the shipboard routines where two apparent dips permitted calculation of the orientation of the fault surface (e.g., Emeis, Robertson, Richter, et al., 1996). The multiple recovery of equivalent intervals at the same site allowed an extensive fault data set to be collected (Kopf and Flecker, 1996) and provided the basis for testing of whether shear failure occurs systematically over the same intervals from hole to hole. If this is the case, it would imply that the sediment physical properties are likely to be laterally persistent and to control fault location.

\section{Undrained Shear Strength Tests on Split Cores}

Undrained shear strength $\left(S_{u}\right)$ was measured in order to estimate the rigidity contrast between different sediment types. The principal measurements were obtained using the ODP motorized, miniature vane shear device. The vane, which consists of blades $\sim 1 \mathrm{~cm}$ across, is inserted $\sim 2 \mathrm{~cm}$ into the split core face and rotated at a constant rate. The strength is determined from the torque reading following the procedures of Boyce (1976; "Physical Properties" section, "Explanatory Notes" chapter, Emeis, Robertson, Richter, et al., 1996). Further shear strength measurements were carried out with a hand-held CL700 pocket penetrometer on intervals too firm or fractured to permit the use of the vane shear device. The cylindrical penetrometer is pushed into the split core $6.4 \mathrm{~mm}$ and provides a measure of the unconfined compressive strength $\left(q_{u}\right)$ of the sediment as a function of penetration resistance. Conversion from unconfined strength to undrained strength requires that the penetrometer readings are multiplied by a constant factor of 49, as outlined elsewhere (e.g., Lee, 1985; American Safety Testing and Materials [ASTM], 1987; Emeis, Robertson, Richter, et al., 1996). These shipboard methods produce estimates of the unconfined shear strength of the tested materials under undrained conditions, and so document the cohesion and stiffness of the sediments with their original sedimentary structure.

\section{Geotechnical Shear Box Tests on Remolded Samples}

Both drained peak $\left(S_{p}\right)$ and residual shear strength $\left(S_{r}\right)$ were determined using a Wykeham Farrance standard geotechnical shear box (Roscoe, 1953). Samples were remolded (slightly above the original water content), squeezed into a cell of stainless steel $(\sim 60 \times 60 \times 30$ mm inner size), and then loaded initially with $1 \mathrm{~kg}$ weight (equivalent to $\sim 44 \mathrm{kPa}$ ). Thereafter, the sample was consolidated with a loading increment ratio of one (following British Standards, 1991; Demars and Chaney, 1992 [ASTM STP 777]) until it reached an equivalent of $\sim 1100 \mathrm{kPa}$. The sample, which was assumed to be normally consolidated, was then sheared at a rate of $0.002 \mathrm{~mm} / \mathrm{min}$ for four days to ensure that failure occurred. Additional shear tests were conducted on some samples after unloading from the peak normal stress to moderate overconsolidation ratios. These laboratory tests measure the fully drained frictional properties of the sediment.

\section{Grain-Size Analysis}

Grain-size analyses were carried out using a Coulter LS 100 particle-size analyzer. This instrument uses a focused laser beam swept at constant velocity across a suspension of $4 \%$ mol sodium hexametaphosphate (Calgon solution) and sediment particles. The backscatter of light from the particles is measured as intensity which is divided into 74 intervals between 0.4 and $900 \mu \mathrm{m}$ of variable width (i.e., $0.045-91.5 \mu \mathrm{m})$. These intervals are allocated to grain-size classes following the grain-size classification outlined by Tucker (1981). The distinction between the clay and silt fraction is made at $2 \mu \mathrm{m}$ and particles $>500 \mu \mathrm{m}$ are counted as the sand component.

\section{Microstructural Analysis}

Samples from the shear-box tests were air dried and divided into chips for thin-section preparation, and small subsamples were glued onto stubs and gold coated for scanning electron microscope (SEM) study.

\section{Organic Geochemistry}

The geochemical results used in this study were generated on board the JOIDES Resolution during Leg 160 (Emeis, Robertson, Richter, et al., 1996). As part of the shipboard routine analyses, total carbon was measured using a Carlo Erba $1500 \mathrm{CNS}$ analyzer. About $5 \mathrm{mg}$ of freeze-dried, ground sediment with an added $\mathrm{V}_{2} \mathrm{O}_{5}$ catalyst were combusted at $\sim 1000^{\circ} \mathrm{C}$ in a stream of oxygen. After reduction of nitrogen oxides, the mixture of $\mathrm{SO}_{2}, \mathrm{CO}_{2}$, and $\mathrm{N}_{2}$ was separated by gas chromatography and quantified with a thermal conductivity detector. Total organic carbon content of the sediment samples was obtained by calculating the difference between carbonate carbon (determined with a Coulometrics 5011 carbonate carbon analyzer) and the total carbon value (Emeis, Robertson, Richter, et al., 1996).

\section{RESULTS}

\section{Structural Data Collected During Leg 160}

Microscale normal faulting was generally found to be more abundant in sapropel-bearing intervals than in the sapropel-free PlioceneQuaternary sediments recovered from the Eastern Mediterranean. In the intervals studied (see Table 1), all the sediment types recovered (i.e., nannofossil ooze, nannofossil clay, sapropels, turbiditic silt) were layered and faulted in places. However, there is evidence for a higher fault abundance in sapropel-bearing intervals (Fig. 3), probably because the contrasts in sediment physical properties between sapropel and other sediment types are more significant than they are between the nonsapropel sediments (e.g., nannofossil ooze and nannofossil clay). In the plane in which the core was split, the distance between a normal fault and the next sapropel above or beneath the fault never exceeded $4 \mathrm{~m}$. On average, the distance between a sapropel bed and the closest fault was $46.3 \mathrm{~cm}$, while over intervals where sapropels are absent, half the distance between two faults is $\sim 1.5 \mathrm{~m}$ (Table 1; see also "Structural Geology" sections in the site chapters of Emeis, Robertson, Richter, et al., 1996). A plot of the abundance of sapropels and faults, shown on a hole-by-hole basis in order to avoid extreme variations on core scale (Fig. 3), illustrates that faults occur predominantly when sapropels are interbedded within the sediment. In contrast, faulting was observed rarely (or not at all) in cores recovered from the same drill sites where sapropels are absent (see "Structural Geology" sections in the site chapters of Emeis, Robertson, Richter, et al., 1996). For comparison, the percentage of faults observed in the sapropel bearing intervals relative to the total number of faults in the sediments recovered with the APC device is given in Figure 3.

Fault dips are generally steep $\left(50^{\circ}-75^{\circ}\right)$ and often intersect sapropel beds. Some of the faults entrain sapropels along their planes (Fig. 2; for more examples, see "Site 966" chapter, Figs. 35 and 36, in Emeis, Robertson, Richter, et al., 1996). All 19 cores selected for this study contain both sapropel horizons and faults. 


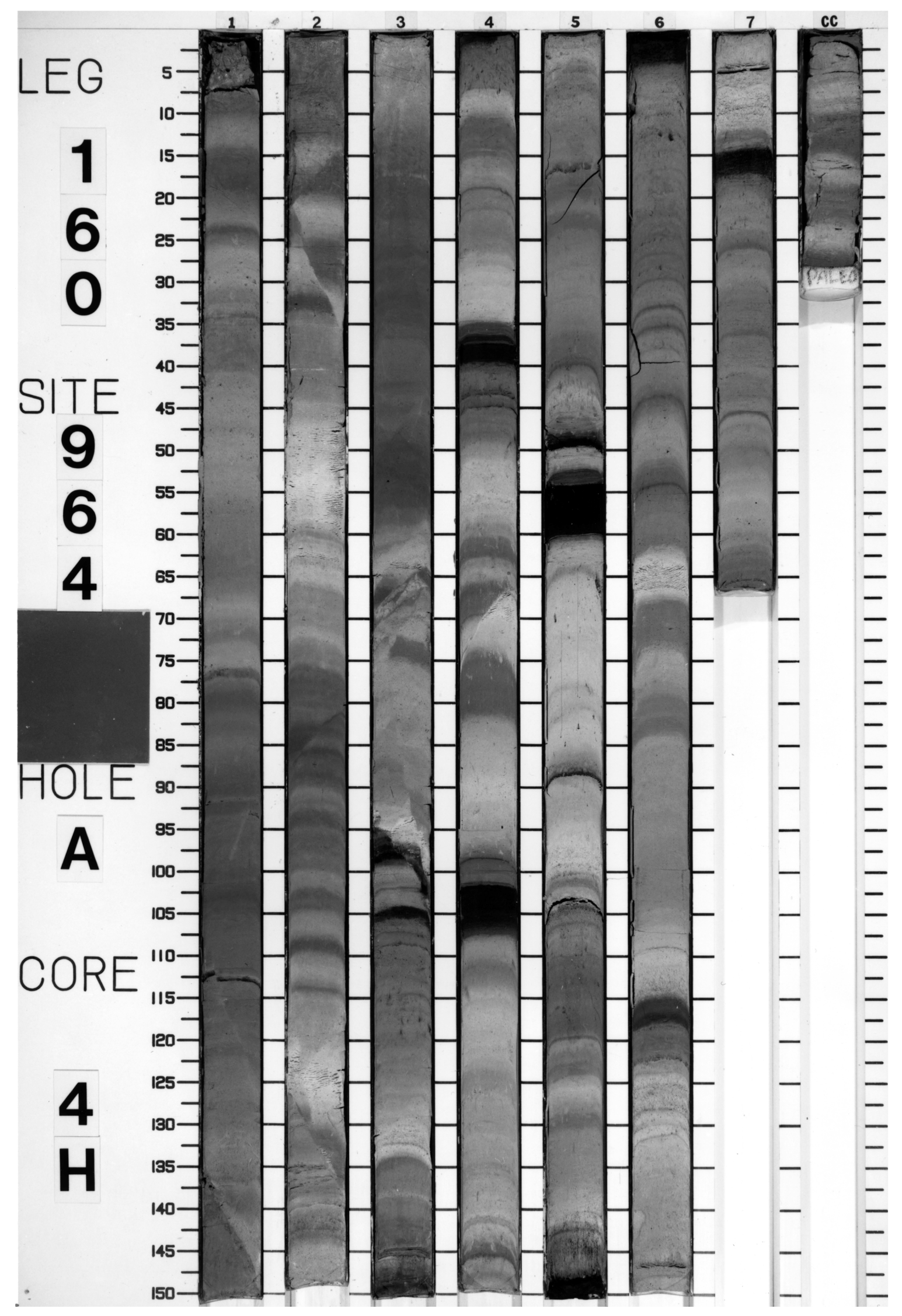

Figure 2. A. Photograph of Core 160-964A-4H. (Continued next page.) 


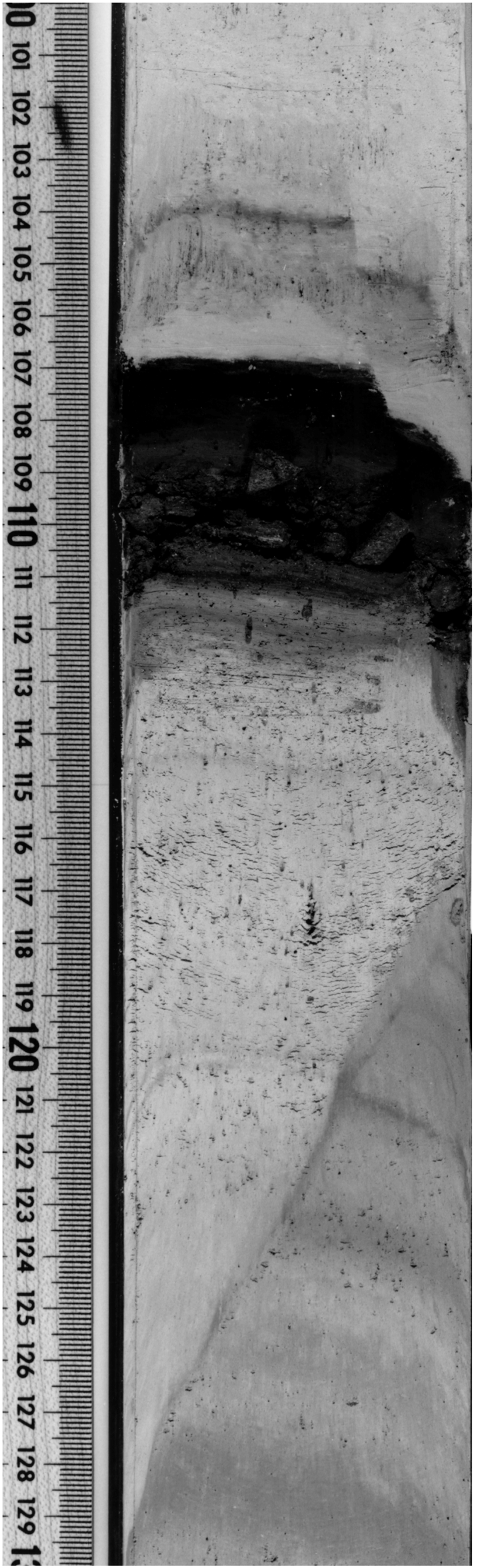

Figure 2 (continued). B. Close-up photograph of interval 160-964A-7H-4, $100-130 \mathrm{~cm}$, showing typical high-angle, normal faulting intersecting a sapropel bed.
Table 1. Number of faults, length of APC-cored section, and averaged half distance between two faults for Sites 964-968.

\begin{tabular}{ccc}
\hline Hole & $\begin{array}{c}\text { Length of APC-cored } \\
\text { interval (m) }\end{array}$ & Number of faults \\
\hline 964A & 101.8 & 47 \\
$964 \mathrm{D}$ & 108.6 & 31 \\
$965 \mathrm{~A}$ & 29.3 & 6 \\
$966 \mathrm{~A}$ & 63.8 & 12 \\
$966 \mathrm{C}$ & 95.1 & 26 \\
$966 \mathrm{D}$ & 84.5 & 23 \\
$967 \mathrm{~A}$ & 123.3 & 24 \\
$967 \mathrm{~B}$ & 119.3 & 56 \\
$967 \mathrm{C}$ & 114.4 & 16 \\
$968 \mathrm{~A}$ & 85 & 82 \\
$\Sigma$ & 925.1 & 323 \\
\hline
\end{tabular}

Notes: Structural data from Emeis, Robertson, Richter, et al. (1996). Note that only the holes where structural measurements on the core were permitted were considered. Calculations: $92510 \mathrm{~cm} / 323$ faults $=286.4 \mathrm{~cm} ; 286.4 / 2=143.1-\mathrm{cm}$ average halfdistance between two faults.
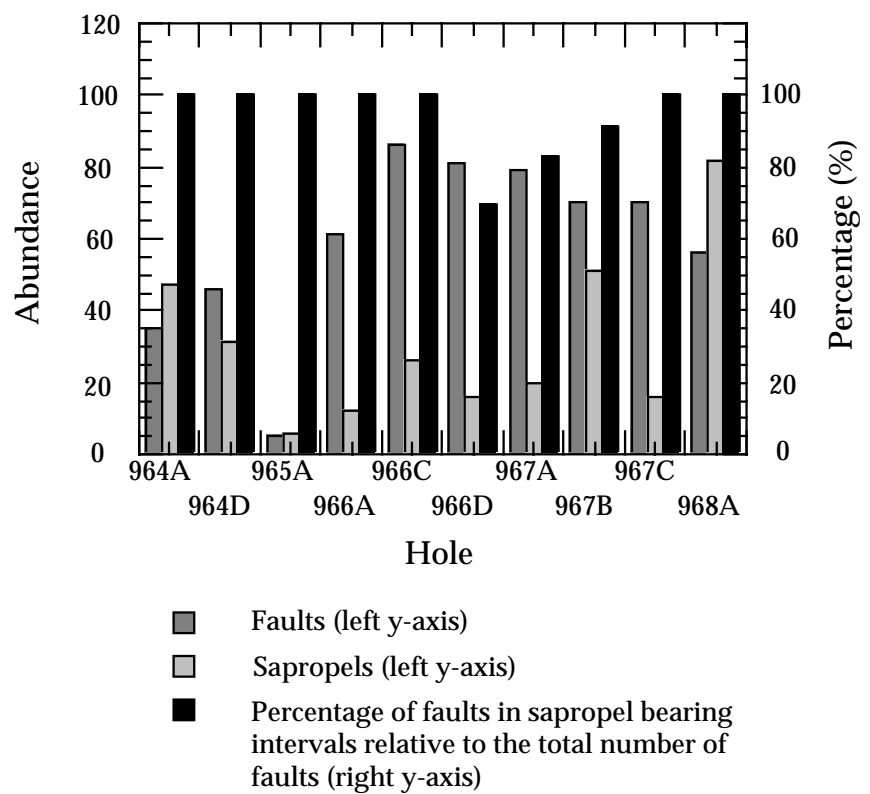

Figure 3. Fault and sapropel abundances in the successions recovered by APC coring, illustrated on a hole-by-hole basis. The right column of each hole shows the number of faults in the intervals regarded (left column of each hole) relative to the total number of faults per hole, given as percentage. See text and Table 4. Note the different units on the two y-axes.

\section{Undrained Shear Strength of Sediments}

Undrained shear strength data vary in response to the rigidity difference between nannofossil ooze, nannofossil clay, and sediment rich in organic carbon (sapropels). The results of shear strength determined with the vane apparatus as part of Leg 160 shipboard routine are illustrated in Figure 4. It should be mentioned that sapropel shear strength was not routinely determined on board the JOIDES Resolution because of the necessity of preserving undisturbed sequences of organic-rich layers for post-cruise sampling. Despite this, some vane shear measurements were obtained from organic-rich layers (Y. Mart, pers. comm., 1995) and provided higher shear strength values than those of the nannofossil oozes (Site 967; below). Pocket penetrometer measurements on a layer-by-layer basis (Table 2) obtained shear strengths on the nannofossil oozes that range in the same order of magnitude as the shipboard vane shear results (Figs. 4, 5). However, higher values were obtained for sapropel layers with the pocket penetrometer than with the vane device.

In addition, closely spaced shear strength measurements clearly permit the variability of sediment response, both to undrained shear 
A
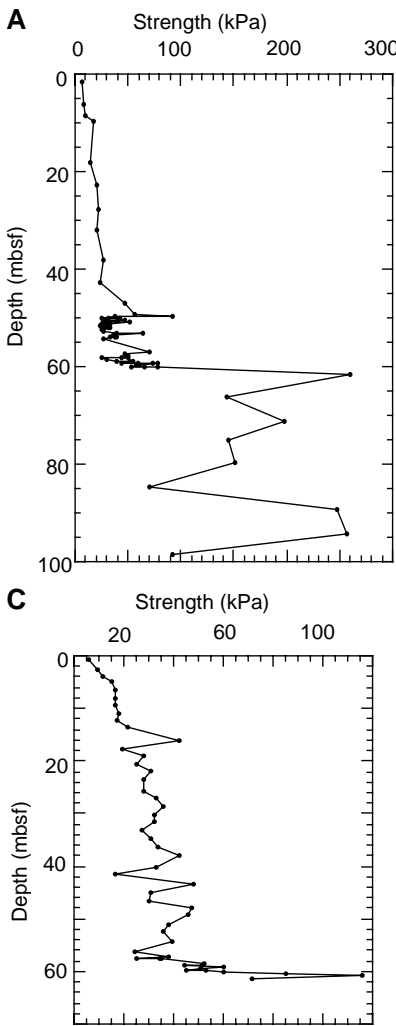

B Strength $(\mathrm{kPa})$

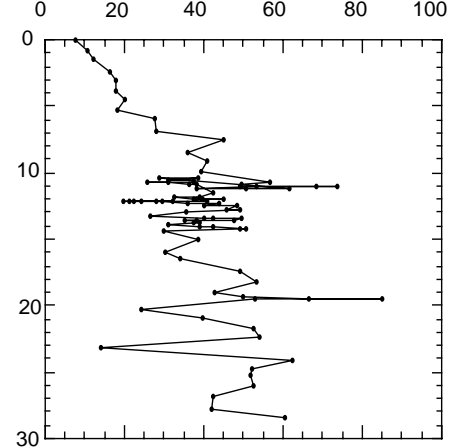

D

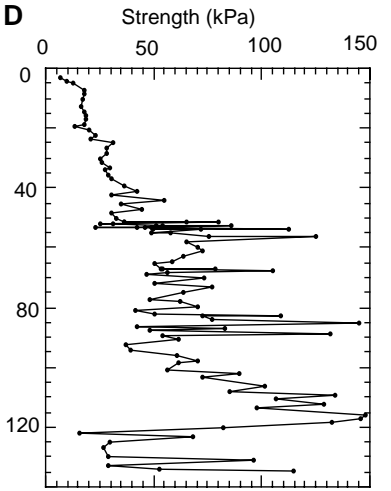

Figure 4. Vane shear strength results from measurements carried out during Leg 160 (Emeis, Robertson, Richter, et al., 1996). A. Site 964. B. Site 965. C. Site 966. D. Site 967. Note that all diagrams vary in scale.

(vane) and unconfined compression (pocket penetrometer) perpendicular to the core axis, to be related to the layered nature of the successions recovered (e.g., Fig. 2A).

In summary, vane shear strength measurements range from 13 $\mathrm{kPa}$ to $50 \mathrm{kPa}$ at the very top of the successions studied to up to $\sim 120$ $\mathrm{kPa}$ at the base of the APC drilled sections (for exact depths in each hole, see Table 2). The maximum values were generally obtained from sapropel layers, ranging from 120 to $150 \mathrm{kPa}$. The majority of the pocket penetrometer data scatters between 15 and $65 \mathrm{kPa}$ for the upper portion of the sediment pile, and values of 50-130 $\mathrm{kPa}$ were observed deeper in the hole (Table 2). Maximum values were obtained from sapropel layers and indurated silt and sand layers, occasionally reaching $>220 \mathrm{kPa}$. Taking all the measurements together, it can be concluded that the pocket penetrometer resulted in a systematic overestimation of sediment strength. Nearly two-thirds of the pocket penetrometer data (65\%; Table 2 ) are significantly higher than the values obtained using the vane apparatus.

The dependence of the shear strength results on the method chosen is also reflected in the average values calculated for each technique and lithology. The mean shear strength of nannofossil ooze and clay for the intervals from Sites $964-968$ is $69 \mathrm{kPa}$ (vane) and $98 \mathrm{kPa}$ (pocket penetrometer). For the sapropels and sapropelic (i.e., organic rich, oxidized, or burnt down) layers, average values range from 73 $\mathrm{kPa}$ for the vane shear apparatus to $109 \mathrm{kPa}$ for the pocket penetrometer (calculated as the mean of 23 sapropel beds; Table 2). Note that these average data are compiled from sediments at variable depths and, therefore, are meaningful only for comparative purposes. Nevertheless, all the intervals chosen (i.e., the 19 cores) have sapropel layers interbedded so that the same depth interval is looked at although measurements in nannofossil ooze were carried out more frequently. Measurements, however, were made fairly regularly in either lithology (Table 1).

\section{Results from Drained Shear Box Tests and Grain-Size Analysis}

Peak and residual shear strengths were determined from a single sapropel and a single nannofossil ooze specimen that were thought to be representative of hemipelagic sedimentation in the region. For the nannofossil ooze, a peak strength of $621 \mathrm{kPa}$ and a residual strength of $<580 \mathrm{kPa}$ were obtained at the maximum normal stress of $\sim 1 \mathrm{MPa}$ (Table 3). Values of $30^{\circ}$ and $<28^{\circ} \pm 1^{\circ}$ for the angles of internal friction were calculated, which are typical for fine-grained carbonates (Demars and Chaney, 1992). These values suggest that carbonate, rather than clay minerals, is the phase-controlling failure (e.g, Arkin, 1986; Arkin and Michaeli, 1989). By comparison, the sapropel specimen (total organic-carbon content of the sapropel layer above $=$ $22.5 \%$, and underneath $=12.6 \%$, but not determined for this particular bed; Emeis, Robertson, Richter, et al., 1996) had a peak drained shear strength of only $253 \mathrm{kPa}$ and a residual drained shear strength less than $226 \mathrm{kPa}$ at $1 \mathrm{MPa}$ normal load (Table 3). The internal angles of friction were $11^{\circ} \pm 1^{\circ}\left(\phi^{\prime} p\right)$ and $<10^{\circ} \pm 1^{\circ}\left(\phi^{\prime} r\right)$. The peak values are very low, typical of weaker clay minerals (i.e., smectite or mixedlayer clays; Skempton, 1985) though without the extremely low residual strength these minerals exhibit.

The microfabrics and textures developed during shearing are different for each lithology (Fig. 6). The nannofossil ooze developed aligned foraminifer tests along the main, central shear zone (between the two halves of the cell of the shear box). In addition, mobile dark material was enriched in the pressure shadows of rotated foraminifer shells (Fig. 7A). From the central shear zone, Riedel shear bands and anastomosing veinlets intersected the relatively homogeneous matrix. In places, these microfractures led to mosaic textures. The width of the central shear zone is $\sim 0.5-0.9 \mathrm{~mm}$, but this can be partly artificial (i.e., a result of thin-section preparation). The slightly brighter material along the shear surfaces is interpreted to be the result either of enhanced compaction accompanying particle alignment, or mobilization of a finer component (e.g., kaolinite, as suggested from XRD studies; Robertson and Kopf, Chap. 45, this volume). Dispersed opaque components, both pyrite and organic matter, are enriched within the shear bands, but can be found occasionally elsewhere in the matrix. Mobilization and segregation of the finer component was more distinctly observed in the sheared sapropel sample (Fig. 7B). Here, the shear zones are clearly marked by enrichment in a light gray component of nannofossil clay and ooze. Bearing in mind that the remolded material was perfectly homogeneous before the test, a considerable amount of particulate flow must have occurred during shearing (e.g., Borradaile, 1981; Knipe, 1986). This result also indicates that even during sapropel formation, hemipelagic background sedimentation of carbonate ooze takes place. Shear zones often form duplex structures and submillimeter folds. In addition, foraminifer tests act as typical $\sigma$-clasts with light nannofossil clay in their "pressure shadows" (Ramsay and Huber, 1987).

These results may be partially explainable by the difference in grain size, which was determined for the two shear box samples (Table 3). Particles within the nannofossil ooze generally do not exceed $200 \mu \mathrm{m}$, whereas sapropels, even though dispersed in Calgon solution for more than $24 \mathrm{hr}$, contain particles exceeding $1 \mathrm{~mm}$ in diameter. During further examination of smear slides under the microscope, however, this sand-size fraction often turned out to be aggregates of finer particles. Organic compounds are considered to cause this particle bonding (Busch and Keller, 1982; see discussion below).

\section{Organic Carbon Content}

No significant differences in organic-carbon content was observed among the sites studied ("Organic Geochemistry" sections in the site chapters of Emeis, Robertson, Richter, et al., 1996). Organic carbon values of the background sediment (i.e., the hemipelagic clays and oozes) generally range between $0.05 \%$ and $0.5 \%$. Some nannofossil oozes, however, had $\mathrm{C}_{\text {org }}$ contents of up to $2 \%$. Sediments re- 
Table 2. High-resolution results of shear strength and pocket penetrometer measurements from Sites 964 to 968.

\begin{tabular}{|c|c|c|c|c|c|c|c|c|}
\hline $\begin{array}{l}\text { Core, section, } \\
\text { interval }(\mathrm{cm})\end{array}$ & $\begin{array}{l}\text { Depth } \\
(\mathrm{mbsf})\end{array}$ & $\begin{array}{l}\text { Strength } \\
(\mathrm{kPa})\end{array}$ & $\begin{array}{cc} & \text { Distance to fault } \\
\text { Sapropel } & (\mathrm{cm})\end{array}$ & $\begin{array}{l}\text { Core, section, } \\
\text { interval }(\mathrm{cm})\end{array}$ & $\begin{array}{l}\text { Depth } \\
\text { (mbsf) }\end{array}$ & $\begin{array}{l}\text { Strength } \\
(\mathrm{kPa})\end{array}$ & Sapropel & $\begin{array}{l}\text { Distance to fault } \\
(\mathrm{cm})\end{array}$ \\
\hline 160-964A- & & & & $3 \mathrm{H}-1,11.6-11.7$ & 10.42 & 38.6 & & \\
\hline $1 \mathrm{H}-2,22.6-22.7$ & 1.73 & 5.6 & & $3 \mathrm{H}-2,18-18.1$ & 10.48 & 30.8 & & \\
\hline $1 \mathrm{H}-5,26.4-26.5$ & 6.26 & 7.4 & & $3 \mathrm{H}-2,26.6-26.7$ & 10.57 & 37.3 & & \\
\hline $2 \mathrm{H}-2,26.9-27$ & 8.57 & 9.3 & & $3 \mathrm{H}-2,32.5-32.6$ & 10.63 & 37.5 & & \\
\hline $2 \mathrm{H}-2,114.2-114.3$ & 9.44 & 16.4 & & $3 \mathrm{H}-1,40.7-40.8$ & 10.71 & 25.6 & & \\
\hline $3 \mathrm{H}-2,26.7-26.8$ & 18.07 & 14 & & $3 \mathrm{H}-2,43.4-43.5$ & 10.73 & 30.8 & & \\
\hline $3 \mathrm{H}-5,26-26.1$ & 22.56 & 20.6 & & $2 \mathrm{H}-7,94-94.1$ & 10.74 & 56.5 & & \\
\hline $4 \mathrm{H}-2,26.8-26.9$ & 27.57 & 22.5 & & $3 \mathrm{H}-1,51.6-51.7$ & 10.82 & 36.2 & & \\
\hline $4 H-5,29.1-29.2$ & 32.09 & 20.9 & & $2 \mathrm{H}-7,109.5-109.6$ & 10.9 & 49.6 & & \\
\hline $5 \mathrm{H}-2,146.5-146.6$ & 38.27 & 26.2 & & $3 \mathrm{H}-1,61.8-61.9$ & 10.92 & 38.3 & & \\
\hline $5 \mathrm{H}-5,128.5-128.6$ & 42.59 & 22.9 & & $2 \mathrm{H}-7,119-119.1$ & 10.99 & 48.9 & & \\
\hline $6 \mathrm{H}-2,52.7-52.8$ & 46.83 & 46.9 & & $2 \mathrm{H}-7,119.9-120$ & 11 & 73.7 & & \\
\hline $6 \mathrm{H}-4,2.9-3$ & 49.329 & 56.1 & & $2 \mathrm{H}-7,125.4-125.5$ & 11.05 & 68.3 & & \\
\hline $6 \mathrm{H}-4,16.1-16.2$ & 49.461 & 91.9 & & $2 \mathrm{H}-7,127-127.1$ & 11.07 & 53.2 & & \\
\hline $6 \mathrm{H}-4,27.7-27.8$ & 49.577 & 37.9 & & $2 \mathrm{H}-7,130.8-130.9$ & 11.11 & 38.3 & & \\
\hline $6 \mathrm{H}-4,53.3-53.4$ & 49.833 & 25.1 & & $2 \mathrm{H}-7,136-136.1$ & 11.16 & 50.4 & & \\
\hline $6 \mathrm{H}-4,65.8-65.9$ & 49.958 & 42.7 & & $2 \mathrm{H}-7,141.1-141.2$ & 11.21 & 61.7 & & \\
\hline $6 \mathrm{H}-4,112.7-112.8$ & 50 & 46 & & $3 \mathrm{H}-1,118.3-118.4$ & 11.48 & 42.4 & & \\
\hline $6 \mathrm{H}-4,87.2-87.3$ & 50.172 & 30.9 & & $3 \mathrm{H}-2,3-3.1$ & 11.83 & 39 & & \\
\hline $6 \mathrm{H}-4,100.3-100.4$ & 50.3 & 28.6 & & $3 \mathrm{H}-2,7.2-7.3$ & 11.872 & 32.4 & & \\
\hline $6 \mathrm{H}-4,134-134.1$ & 50.64 & 29.4 & & $3 \mathrm{H}-2,9.9-10$ & 11.899 & 37.4 & & \\
\hline $6 \mathrm{H}-4,139.4-139.5$ & 50.69 & 25.8 & & $3 \mathrm{H}-2,16.1-16.2$ & 11.961 & 39.8 & & \\
\hline $6 \mathrm{H}-4,146.8-146.9$ & 50.77 & 26 & & $3 \mathrm{H}-2,21-21.1$ & 12.01 & 45 & & \\
\hline $6 \mathrm{H}-5,9.5-9.6$ & 50.9 & 52 & & $3 \mathrm{H}-2,21.1-21.2$ & 12.011 & 38.1 & & \\
\hline $6 \mathrm{H}-4,123.5-123.6$ & 51 & 40.7 & & $3 \mathrm{H}-2,25.4-25.5$ & 12.054 & 40.6 & & \\
\hline $6 \mathrm{H}-5,26.5-26.6$ & 51.07 & 32.7 & & $3 \mathrm{H}-2,28-28.1$ & 12.08 & 22.4 & & \\
\hline $6 \mathrm{H}-5,37.2-37.3$ & 51.17 & 24.7 & & $3 \mathrm{H}-2,29-29.1$ & 12.09 & 19.5 & & \\
\hline $6 \mathrm{H}-5,50.4-50.5$ & 51.3 & 29.3 & & $3 \mathrm{H}-2,32-32.1$ & 12.12 & 28 & & \\
\hline $6 \mathrm{H}-5,68.5-68.6$ & 51.49 & 22.9 & & $3 \mathrm{H}-2,32.8-32.9$ & 12.13 & 31.9 & & \\
\hline $6 \mathrm{H}-5,76.5-76.6$ & 51.57 & 37.6 & & $3 \mathrm{H}-2,35-35.1$ & 12.15 & 21.2 & & \\
\hline $6 \mathrm{H}-5,100-100.1$ & 51.8 & 32.7 & & $3 \mathrm{H}-2,40-40.1$ & 12.2 & 24.1 & & \\
\hline $6 \mathrm{H}-5,116.9-117$ & 51.97 & 31.1 & & $3 \mathrm{H}-2,40.6-40.7$ & 12.206 & 29.6 & & \\
\hline $6 \mathrm{H}-5,129.9-130$ & 52.1 & 27.4 & & $3 \mathrm{H}-2,46.8-46.9$ & 12.268 & 35.7 & & \\
\hline $6 \mathrm{H}-6,15.2-15.3$ & 52.45 & 24.8 & & $3 \mathrm{H}-2,52.9-53$ & 12.329 & 43.8 & & \\
\hline $6 \mathrm{H}-6,43.3-43.4$ & 52.73 & 26.9 & & $3 \mathrm{H}-2,62.4-62.5$ & 12.424 & 39.9 & & \\
\hline $6 \mathrm{H}-6,70.1-70.2$ & 53 & 39 & & $3 \mathrm{H}-2,66.7-66.8$ & 12.47 & 48.3 & & \\
\hline $6 \mathrm{H}-6,86.5-86.6$ & 53.17 & 64.5 & & $3 \mathrm{H}-2,112.8-112.9$ & 12.93 & 35.4 & & \\
\hline $6 \mathrm{H}-6,106.3-106.4$ & 53.36 & 35.5 & & $3 \mathrm{H}-2,100.1-100.2$ & 13 & 48.9 & & \\
\hline $6 \mathrm{H}-6,146.4-146.5$ & 53.76 & 33.1 & & $3 \mathrm{H}-2,90.2-90.3$ & 13 & 45.7 & & \\
\hline $6 \mathrm{H}-7,1.8-1.9$ & 53.82 & 38.5 & & $3 \mathrm{H}-3,2.2-2.3$ & 13.322 & 26.3 & & \\
\hline $6 \mathrm{H}-7,4.1-4.2$ & 53.84 & 36.7 & & $3 \mathrm{H}-3,5.3-5.4$ & 13.353 & 40 & & \\
\hline $6 \mathrm{H}-7,11.7-11.8$ & 53.92 & 37.4 & & $3 \mathrm{H}-3,7.5-7.6$ & 13.375 & 42.4 & & \\
\hline $6 \mathrm{H}-7,29.9-30$ & 54.1 & 25.8 & & $3 \mathrm{H}-3,13.7-13.8$ & 13.437 & 49.4 & & \\
\hline $7 \mathrm{H}-2,116.2-116.3$ & 56.96 & 70.6 & & $3 \mathrm{H}-3,20.6-20.7$ & 13.506 & 47.6 & & \\
\hline $7 \mathrm{H}-3,12.8-12.9$ & 57.428 & 46.9 & & $3 \mathrm{H}-3,25-25.1$ & 13.55 & 35.2 & & \\
\hline $7 \mathrm{H}-3,22.1-22.2$ & 57.521 & 49.8 & & $3 \mathrm{H}-3,28.6-28.7$ & 13.586 & 38 & & \\
\hline $7 \mathrm{H}-3,35.9-36$ & 57.659 & 49.5 & & $3 \mathrm{H}-3,36.2-36.3$ & 13.662 & 37.4 & & \\
\hline $6 \mathrm{H}-4,123.7-123.8$ & 47.84 & 12.9 & & $3 \mathrm{H}-3,46.3-46.4$ & 13.763 & 38.8 & & \\
\hline $6 \mathrm{H}-4,139.1-139.2$ & 47.99 & 30.2 & & $3 \mathrm{H}-3,59.8-59.9$ & 13.898 & 30.9 & & \\
\hline $6 \mathrm{H}-4,145.8-145.9$ & 48.06 & 26.6 & & $3 \mathrm{H}-3,69.7-69.8$ & 13.997 & 39 & & \\
\hline $6 \mathrm{H}-5,11.6-11.7$ & 48.22 & 24.9 & & $3 \mathrm{H}-3,77.3-77.4$ & 14.073 & 42.2 & & \\
\hline $6 \mathrm{H}-5,31.4-31.5$ & 48.41 & 21.6 & & $3 \mathrm{H}-3,82.4-82.5$ & 14.124 & 50.4 & & \\
\hline $6 \mathrm{H}-5,49.9-50$ & 48.6 & 19.3 & & 3H-3, 89.8-89.9 & 14.198 & 48.9 & & \\
\hline $6 \mathrm{H}-5,69.9-70$ & 48.8 & 18.1 & & $3 \mathrm{H}-3,112.7-112.8$ & 14.43 & 29.8 & & \\
\hline $6 \mathrm{H}-5,81.2-81.3$ & 48.91 & 14.6 & & $3 \mathrm{H}-4,25.5-25.6$ & 15.06 & 38.6 & & \\
\hline $6 \mathrm{H}-5,87.2-87.3$ & 48.97 & 15.6 & & $3 \mathrm{H}-4,115.6-115.7$ & 15.96 & 30.2 & & \\
\hline $6 \mathrm{H}-5,110.4-110.5$ & 49.2 & 17.3 & & $3 \mathrm{H}-5,17.4-17.5$ & 16.47 & 33.8 & & \\
\hline $6 \mathrm{H}-5,115.8-115.9$ & 49.26 & 21 & & $3 \mathrm{H}-5,106.4-106.5$ & 17.36 & 49 & & \\
\hline $6 \mathrm{H}-5,128.6-128.7$ & 49.39 & 28.6 & & $3 \mathrm{H}-6,45.9-46$ & 18.26 & 53.1 & & \\
\hline $6 \mathrm{H}-7,11.9-12$ & 51.22 & 23.5 & & $3 \mathrm{H}-6,113.9-114$ & 18.94 & 42.5 & & \\
\hline $6 \mathrm{H}-7,26.9-27$ & 51.37 & 24.4 & & $3 \mathrm{H}-7,2-2.1$ & 19.32 & 49.9 & & \\
\hline $6 \mathrm{H}-7,44.8-44.9$ & 51.55 & 27.3 & & $3 \mathrm{H}-7,16.8-16.9$ & 19.47 & 66.6 & & \\
\hline $6 \mathrm{H}-7,57-57.1$ & 51.67 & 19.9 & & $3 \mathrm{H}-7,18-18.1$ & 19.48 & 84.8 & & \\
\hline $7 \mathrm{H}-1,16.5-16.6$ & 54.465 & 19.3 & & $3 \mathrm{H}-7,24.8-24.9$ & 19.55 & 53 & & \\
\hline $7 \mathrm{H}-1,58.5-58.6$ & 54.885 & 26.4 & & $4 \mathrm{H}-1,39.8-39.9$ & 20.198 & 24.1 & & \\
\hline $7 \mathrm{H}-1,73-73.1$ & 55.03 & 33 & & $4 \mathrm{H}-1,112.2-112.3$ & 20.922 & 39.7 & & \\
\hline $7 \mathrm{H}-1,81.4-81.5$ & 55.114 & 30.4 & & $4 \mathrm{H}-2,41.5-41.6$ & 21.715 & 52.3 & & \\
\hline $7 \mathrm{H}-1,87.9-88$ & 55.179 & 28.7 & & $4 \mathrm{H}-2,106.4-106.5$ & 22.364 & 53.9 & & \\
\hline $7 \mathrm{H}-1,127.3-127.4$ & 55.573 & 28.4 & & $4 \mathrm{H}-3,32.8-32.9$ & 23.128 & 13.8 & & \\
\hline $7 \mathrm{H}-1,132.1-132.2$ & 55.621 & 30.2 & & $4 \mathrm{H}-3,136.3-136.4$ & 24.163 & 62.4 & & \\
\hline $7 \mathrm{H}-1,139-139.1$ & 55.69 & 25.1 & & $4 \mathrm{H}-4,45.2-45.3$ & 24.752 & 52.2 & & \\
\hline $160-0654$ & & & & $4 \mathrm{H}-4,94.3-94.4$ & 25.243 & 51.6 & & \\
\hline 160-965A- & & & & $4 \mathrm{H}-5,20.9-21$ & 26.009 & 52.4 & & \\
\hline $1 \mathrm{H}-1,4.2-4.3$ & 0.04 & $\begin{array}{r}7.5 \\
10.6\end{array}$ & & $4 \mathrm{H}-5,101.2-101.3$ & 26.812 & 42.1 & & \\
\hline $2 \mathrm{H}-1,7.1-7.2$ & 0.87 & 10.6 & & $4 \mathrm{H}-6,44.5-44.6$ & 27.745 & 41.8 & & \\
\hline $\begin{array}{l}2 \mathrm{H}-1,62.2-62.3 \\
2 \mathrm{H}-2,4.1-4.2\end{array}$ & $\begin{array}{l}1.42 \\
2.34\end{array}$ & $\begin{array}{l}12.1 \\
16.2\end{array}$ & & $4 \mathrm{H}-6,109.6-109.7$ & 28.396 & 60.3 & & \\
\hline $\begin{array}{l}2 \mathrm{H}-2,4.1-4.2 \\
2 \mathrm{H}-2,81.1-81.2\end{array}$ & $\begin{array}{l}2.34 \\
3.11\end{array}$ & $\begin{array}{l}16.2 \\
17.8\end{array}$ & & $160-966 \mathrm{D}-$ & & & & \\
\hline $2 \mathrm{H}-3,8.8-8.9$ & 3.89 & 17.8 & & $6 \mathrm{H}-2,39.2-39.3$ & 43.39 & 34 & & \\
\hline $2 \mathrm{H}-3,70.1-70.2$ & 4.5 & 20.2 & & $6 \mathrm{H}-2,63.6-63.7$ & 43.64 & 33.8 & & \\
\hline $2 \mathrm{H}-4,3.2-3.3$ & 5.33 & 18 & & $6 \mathrm{H}-2,80.2-80.3$ & 43.8 & 38.9 & & \\
\hline $2 \mathrm{H}-4,63.1-63.2$ & 5.93 & 27.6 & & $6 \mathrm{H}-2,89.3-89.4$ & 43.89 & 33.5 & & \\
\hline $2 \mathrm{H}-5,64.5-64.6$ & 7.45 & 44.9 & & $6 \mathrm{H}-2,129.7-129.8$ & 44.3 & 26.7 & & \\
\hline $2 \mathrm{H}-6,8.4-8.5$ & 8.38 & 35.9 & & $6 \mathrm{H}-2,143.3-143.4$ & 44.43 & 20.5 & & \\
\hline $2 \mathrm{H}-6,79.5-79.6$ & 9.1 & 40.7 & & $6 \mathrm{H}-3,10.6-10.7$ & 44.61 & 25 & & \\
\hline $2 \mathrm{H}-7,4.9-5$ & 9.85 & 39.1 & & $6 \mathrm{H}-3,22.9-23$ & 44.73 & 17.2 & & \\
\hline $3 \mathrm{H}-1,5.2-5.3$ & 10.35 & 28.6 & & $6 \mathrm{H}-3,40.2-40.3$ & 44.9 & 27.3 & & \\
\hline & & & & $6 \mathrm{H}-3,56.3-56.4$ & 45.06 & 22.4 & & \\
\hline & & & & $6 \mathrm{H}-3,89-89.1$ & 45.39 & 31.5 & & \\
\hline
\end{tabular}


Table 2 (continued).

\begin{tabular}{|c|c|c|c|c|c|c|c|c|}
\hline $\begin{array}{l}\text { Core, section, } \\
\text { interval }(\mathrm{cm})\end{array}$ & $\begin{array}{l}\text { Depth } \\
\text { (mbsf) }\end{array}$ & $\begin{array}{l}\text { Strength } \\
(\mathrm{kPa})\end{array}$ & Sapropel & $\begin{array}{l}\text { Distance to fault } \\
(\mathrm{cm})\end{array}$ & $\begin{array}{l}\text { Core, section, } \\
\text { interval }(\mathrm{cm})\end{array}$ & $\begin{array}{l}\text { Depth } \\
\text { (mbsf) }\end{array}$ & $\begin{array}{l}\text { Strength } \\
(\mathrm{kPa})\end{array}$ & Sapropel \\
\hline $6 \mathrm{H}-3,108.3-108.4$ & 45.58 & 20.2 & & & $10 \mathrm{H}-2,39.4-39.5$ & 87.194 & 34.4 & \\
\hline $6 \mathrm{H}-3,127.3-127.4$ & 45.77 & 36.2 & & & $10 \mathrm{H}-3,33-33.1$ & 88.63 & 38.4 & \\
\hline $6 \mathrm{H}-3,139.6-139.7$ & 45.9 & 32.4 & & & $10 \mathrm{H}-4,27-27.1$ & 90.07 & 44 & \\
\hline $7 \mathrm{H}-3,72-72.1$ & 54.72 & 32.1 & & & $10 \mathrm{H}-5,42.3-42.4$ & 91.723 & 26.6 & \\
\hline $7 \mathrm{H}-3,101.9-102$ & 55.02 & 34.7 & & & $10 \mathrm{H}-6,56-56.4$ & 93.36 & 27.8 & \\
\hline $7 \mathrm{H}-3,139.3-139.4$ & 55.39 & 42 & & & $11 \mathrm{H}-1,68-68.2$ & 95.48 & 43.4 & \\
\hline $160-967 \mathrm{~A}-$ & & & & & $11 \mathrm{H}-2,71-71.4$ & 97.01 & 50.5 & \\
\hline $1 \mathrm{H}-2,84-84.2$ & 2.34 & 5 & & & $11 \mathrm{H}-3,26.8-26.9$ & 98.068 & 43.7 & \\
\hline $1 \mathrm{H}-3,65-65.1$ & 3.65 & 6.7 & & & $11 \mathrm{H}-4,69-69.1$ & 99.99 & 40.2 & \\
\hline $1 \mathrm{H}-4,5.4-5.5$ & $\begin{array}{l}3.05 \\
4.55\end{array}$ & $\begin{array}{l}6.7 \\
9.1\end{array}$ & & & $11 \mathrm{H}-5,43.7-43.8$ & 101.237 & 64.3 & \\
\hline $1 \mathrm{H}-5,66-66.3$ & 6.66 & $\begin{array}{r}9.1 \\
12.4\end{array}$ & & & $11 \mathrm{H}-6,35.6-35.7$ & 102.656 & 51.8 & \\
\hline $1 \mathrm{H}-6,27.6-27.7$ & 7.78 & 12.8 & & & $12 \mathrm{H}-1,127-126.9$ & 105.57 & 72.6 & \\
\hline $2 \mathrm{H}-1,33.4-33.5$ & 9.63 & 12.4 & & & $12 \mathrm{H}-2,141-140.9$ & 107.21 & 60.7 & \\
\hline $2 \mathrm{H}-2,146-146.5$ & 12.26 & 11.5 & & & $12 \mathrm{H}-3,126-126.3$ & 108.56 & 95.7 & \\
\hline $2 \mathrm{H}-3,139-139.4$ & $\begin{array}{l}13.20 \\
13.69\end{array}$ & 12.8 & & & $12 \mathrm{H}-4,130-129.8$ & 110.1 & 76.1 & \\
\hline $2 \mathrm{H}-4,139-139.5$ & 15.19 & $\begin{array}{l}13.8 \\
13.3\end{array}$ & & & $12 \mathrm{H}-5,128-127.9$ & 111.58 & 92.1 & \\
\hline $2 \mathrm{H}-5,135-134.8$ & 16.65 & 13.4 & & & $12 \mathrm{H}-6,94-94.2$ & 112.74 & 70.1 & \\
\hline $2 \mathrm{H}-7,48-48.3$ & 18.78 & $\begin{array}{r}15.4 \\
9.7\end{array}$ & & & $13 \mathrm{H}-1,138-138.1$ & 115.18 & 105.9 & \\
\hline $2 \mathrm{H}-6,137-137.3$ & 18.17 & 12.4 & & & $13 \mathrm{H}-2,120-120.1$ & 116.5 & 104 & \\
\hline $3 \mathrm{H}-1,144-144$ & 20.24 & 14.4 & & & $13 \mathrm{H}-3,94-94.4$ & 117.74 & 94.9 & \\
\hline $3 \mathrm{H}-2,126-125.6$ & 21.56 & $\begin{array}{l}16.4 \\
16.2\end{array}$ & & & $13 \mathrm{H}-4,147-146.7$ & 119.77 & 58.5 & \\
\hline $3 \mathrm{H}-3,135-135.2$ & 23.15 & $\begin{array}{l}10.2 \\
14.9\end{array}$ & & & $13 \mathrm{H}-5,134-133.8$ & 121.14 & 11.2 & \\
\hline $3 \mathrm{H}-4,62-61.9$ & 23.92 & 22.4 & & & $13 \mathrm{H}-6,130-129.8$ & 122.6 & 48.8 & \\
\hline $3 \mathrm{H}-5,129-128.8$ & 26.09 & 20 & & & $160-967 \mathrm{C}-$ & & & \\
\hline $3 \mathrm{H}-6,139-138.6$ & 27.69 & 19.9 & & & $6 \mathrm{H}-4,18.2-18.3$ & 52.182 & 30.6 & \\
\hline $4 \mathrm{H}-1,140-140$ & 29.7 & 17.9 & & & $6 \mathrm{H}-4,34.8-34.9$ & 52.348 & 33.8 & \\
\hline $4 \mathrm{H}-2,104-103.9$ & 30.84 & 18.8 & & & $6 \mathrm{H}-4,45-45.5$ & 52.45 & 53 & \\
\hline $4 \mathrm{H}-3,129-128.6$ & 32.59 & 21.3 & & & $6 \mathrm{H}-4,52-52.3$ & 52.52 & 37.6 & \\
\hline $4 \mathrm{H}-4,35.6-35.7$ & 33.16 & 19.7 & & & $6 \mathrm{H}-4,65-65.4$ & 52.65 & 50.9 & \\
\hline $4 \mathrm{H}-5,106-105.9$ & 35.36 & 20.6 & & & $6 \mathrm{H}-4,72-71.7$ & 52.72 & 63.2 & \\
\hline $4 \mathrm{H}-6,44.7-44.8$ & 36.25 & 21.9 & & & $6 \mathrm{H}-4,81-81.2$ & 52.81 & 69.3 & \\
\hline $5 \mathrm{H}-1,105-105.3$ & 38.85 & 25.8 & & & $6 \mathrm{H}-4,87-87.2$ & 52.87 & 51.1 & \\
\hline $5 \mathrm{H}-2,98-98.2$ & 40.28 & 30 & & & $6 \mathrm{H}-4,98-97.6$ & 52.98 & 55.2 & \\
\hline $5 \mathrm{H}-3,109-108.7$ & 41.89 & 21.5 & & & $6 \mathrm{H}-4,103-102.8$ & 53.03 & 63.5 & \\
\hline $5 \mathrm{H}-4,105-105.4$ & 43.35 & 39.2 & & & $6 \mathrm{H}-4,109-109.5$ & 53.09 & 49.1 & \\
\hline $5 \mathrm{H}-5,115-115.4$ & 44.95 & 24.8 & & & $6 \mathrm{H}-4,121-121.5$ & 53.21 & 64.3 & \\
\hline $5 \mathrm{H}-6,136-136.3$ & 46.66 & 31.7 & & & $6 \mathrm{H}-4,127-127$ & 53.27 & 49.7 & \\
\hline $6 \mathrm{H}-1,73-73.3$ & 48.03 & 21.7 & & & $6 \mathrm{H}-4,131-130.6$ & 53.31 & 87.5 & \\
\hline $6 \mathrm{H}-2,78-78$ & 49.58 & 23.5 & & & $6 \mathrm{H}-4,137-136.6$ & 53.37 & 45.4 & \\
\hline $6 \mathrm{H}-3,17.4-17.5$ & 50.47 & 25.7 & & & $6 \mathrm{H}-4,145-145.3$ & 53.45 & 45.4 & \\
\hline $6 \mathrm{H}-3,38-38.1$ & 50.68 & 46.8 & & & $6 \mathrm{H}-5,2-2.1$ & 53.52 & 43.3 & \\
\hline $6 \mathrm{H}-3,54-54.4$ & 50.84 & 25.8 & & & $6 \mathrm{H}-5,12.1-12.2$ & 53.621 & 69 & \\
\hline $6 \mathrm{H}-3,74-74.4$ & 51.04 & 57.2 & & & $6 \mathrm{H}-5,20.4-20.5$ & 53.704 & 51.8 & \\
\hline $6 \mathrm{H}-3,102-102.4$ & 51.32 & 17.8 & & & $6 \mathrm{H}-5,27.7-27.8$ & 53.777 & 65.9 & \\
\hline $6 \mathrm{H}-3,114-113.7$ & 51.44 & 22 & & & $6 \mathrm{H}-5,37.1-37.2$ & 53.871 & 58.4 & \\
\hline $6 \mathrm{H}-3,142-142.5$ & 51.72 & 38.8 & & & $6 \mathrm{H}-5,52-51.7$ & 54.02 & 47.5 & \\
\hline $6 \mathrm{H}-4,18.7-18.8$ & 51.99 & 36.7 & & & $6 \mathrm{H}-5,60-60.4$ & 54.1 & 44.9 & \\
\hline $6 \mathrm{H}-4,40.7-40.8$ & 52.21 & 61.4 & & & & & & \\
\hline $6 \mathrm{H}-4,48-48.5$ & 52.28 & 30.4 & & & $\begin{array}{l}100-908 \mathrm{~A}- \\
4 \mathrm{H}-1,11-11.1\end{array}$ & & & \\
\hline $6 \mathrm{H}-4,61-60.8$ & 52.41 & 16.2 & & & $\begin{array}{l}4 \mathrm{H}-1,11-11.1 \\
4 \mathrm{H}-1,32.6-32.7\end{array}$ & $\begin{array}{l}28.11 \\
28.326\end{array}$ & $\begin{array}{l}14.5 \\
67.7\end{array}$ & \\
\hline $6 \mathrm{H}-4,82-82.3$ & 52.62 & 39.5 & & & $\begin{array}{l}4 \mathrm{H}-1,32.6-32.7 \\
4 \mathrm{H}-1,53.2-53.3\end{array}$ & $\begin{array}{l}28.326 \\
28.532\end{array}$ & $\begin{array}{l}67.7 \\
84.4\end{array}$ & \\
\hline $6 \mathrm{H}-4,93-93.4$ & 52.73 & 34.8 & & & $\begin{array}{l}4 \mathrm{H}-1,3.253 .3 \\
4 \mathrm{H}-1,62.1-62.2\end{array}$ & 28.621 & $\begin{array}{l}84.4 \\
84.4\end{array}$ & \\
\hline $6 \mathrm{H}-4,106-105.9$ & 52.86 & 32.7 & & & $\begin{array}{l}4 \mathrm{H}-1,62.1-62.2 \\
4 \mathrm{H}-1,671-67.2\end{array}$ & 28.671 & $\begin{array}{l}0.4 \\
92.3\end{array}$ & \\
\hline $6 \mathrm{H}-4,131-131.2$ & 53.11 & 80.3 & & & $\begin{array}{l}4 \mathrm{H}-1,67.1-67.2 \\
4 \mathrm{H}-1,764-76.5\end{array}$ & $\begin{array}{l}28.071 \\
28.764\end{array}$ & 63.5 & \\
\hline $6 \mathrm{H}-4,141-140.8$ & 53.21 & 35.6 & & & $4 \mathrm{H}-1,76.4-76.5$ & 28.04 & 0.3 & \\
\hline $6 \mathrm{H}-5,65-64.7$ & 54 & 51.2 & & & $4 \mathrm{H}-1,89.2-89.3$ & 28.892 & 65.4 & \\
\hline $6 \mathrm{H}-5,119-118.7$ & 54.49 & 34.8 & & & $4 \mathrm{H}-1,108.1-108.2$ & 29.081 & 60.4 & \\
\hline & 54.56 & 34.9 & & & $4 \mathrm{H}-1,138.2-138.3$ & 29.382 & 64 & \\
\hline $6 \mathrm{H}-5,133-132.7$ & 54.63 & 41.6 & & & $4 \mathrm{H}-2,22.1-22.2$ & 29.721 & 69.2 & \\
\hline $6 \mathrm{H}-6,73-73.3$ & 55.53 & 53.8 & & & $4 \mathrm{H}-2,39.2-39.3$ & 29.892 & 68.2 & \\
\hline $6 \mathrm{H}-6,110-110.3$ & 56 & 89.4 & & & $4 \mathrm{H}-2,60.5-60.6$ & 30.105 & 67 & \\
\hline $7 \mathrm{H}-1,72-72$ & 57.52 & $\begin{array}{l}0.4 \\
46.7\end{array}$ & & & $4 \mathrm{H}-2,92.6-92.7$ & 30.426 & 55.1 & \\
\hline $7 \mathrm{H}-2,82-81.9$ & 59.12 & $\begin{array}{l}40.1 \\
50.3\end{array}$ & & & $4 \mathrm{H}-2,113.3-113.4$ & 30.633 & 58.8 & \\
\hline $7 \mathrm{H}-3,85-85.4$ & 60.65 & 51.7 & & & $4 \mathrm{H}-2,132.4-132.5$ & 30.824 & 56.1 & \\
\hline $7 \mathrm{H}-4,68-67.6$ & 61.98 & 45.6 & & & $4 \mathrm{H}-3,52.9-53$ & 31.529 & 72.4 & \\
\hline $7 \mathrm{H}-5,108-107.6$ & 63.88 & 42 & & & $4 \mathrm{H}-3,68.2-68.3$ & 31.682 & 71.9 & \\
\hline $7 \mathrm{H}-6,47-47.2$ & 64.77 & 36.2 & & & $4 \mathrm{H}-3,90.30-90.4$ & 31.903 & 59.7 & \\
\hline $8 \mathrm{H}-1,6.1-6.2$ & 66.361 & 38.2 & & & $4 \mathrm{H}-3,110-110.1$ & 32.1 & 71.9 & \\
\hline 8H-1, 26.1-1-26.2 & $\begin{array}{l}00.501 \\
66.561\end{array}$ & $\begin{array}{l}3.2 \\
55.9\end{array}$ & & & $4 \mathrm{H}-3,126.5-126.6$ & 32.265 & 77.7 & \\
\hline $\begin{array}{l}8 \mathrm{H}-1,20.1-20.2 \\
8 \mathrm{H}-1,38.7-38.8\end{array}$ & $\begin{array}{l}00.501 \\
66687\end{array}$ & $\begin{array}{l}5.9 \\
386\end{array}$ & & & $4 \mathrm{H}-3,139.4-139.5$ & 32.394 & 80.5 & \\
\hline $\begin{array}{l}8 \mathrm{H}-1,1,38.7-38.8 \\
8 \mathrm{H}-1,90-89.6\end{array}$ & $\begin{array}{l}66.687 \\
672\end{array}$ & 38.6 & & & $4 \mathrm{H}-3,147.8-147.9$ & 32.478 & 44.4 & \\
\hline $8 \mathrm{H}-1,112-112.4$ & $\begin{array}{l}01.2 \\
67.42\end{array}$ & 40.1 & & & $4 \mathrm{H}-4,36.5-36.6$ & 32.865 & 78.3 & \\
\hline $8 \mathrm{H}-2,20.6-20.7$ & 68.006 & 33.6 & & & $4 \mathrm{H}-4,55.8-55.9$ & 33.058 & 74 & \\
\hline $8 \mathrm{H}-3,2.6-32.7$ & 69.626 & 52.5 & & & $4 \mathrm{H}-4,66.7-66.8$ & 33.167 & 78.5 & \\
\hline $8 \mathrm{H}-4,37.6-37.7$ & $\begin{array}{l}09.020 \\
71.176\end{array}$ & 36.1 & & & $4 \mathrm{H}-4,96.7-96.8$ & 33.467 & 71.2 & \\
\hline $8 \mathrm{H}-5,47-46.8$ & 72.77 & $\begin{array}{l}30.1 \\
55.1\end{array}$ & & & $4 \mathrm{H}-4,117.7-117.8$ & 33.677 & 73.1 & \\
\hline $8 \mathrm{H}-6,42.9-43$ & 74.229 & 45.4 & & & $4 \mathrm{H}-4,121-121.1$ & 33.71 & 86.5 & \\
\hline $9 \mathrm{H}-1,98-98.2$ & 76.78 & 34.2 & & & $4 \mathrm{H}-5,25-25.1$ & 34.25 & 91.2 & \\
\hline $9 \mathrm{H}-2,20.2-20.3$ & 77.502 & 44.7 & & & $4 \mathrm{H}-5,57.4-57.5$ & 34.574 & 103.5 & \\
\hline $9 \mathrm{H}-3,59-58.8$ & 79.39 & 50.5 & & & $4 \mathrm{H}-5,110.3-110.4$ & 35.103 & 73.8 & \\
\hline $9 \mathrm{H}-4,94-93.7$ & 81.24 & $\begin{array}{l}30.5 \\
36\end{array}$ & & & $4 \mathrm{H}-5,128.6-128.7$ & 35.286 & 85.7 & \\
\hline $\begin{array}{l}9 \mathrm{H}-4,94-94.1 \\
9 \mathrm{H}-4,24-24.1\end{array}$ & $\begin{array}{l}81.24 \\
80.54\end{array}$ & $\begin{array}{l}36 \\
29.8\end{array}$ & & & $4 \mathrm{H}-5,144.6-144.7$ & 35.446 & 80.3 & \\
\hline $9 \mathrm{H}-5,60-59.6$ & 82.4 & 516 & & & $5 \mathrm{H}-1,17.6-17.7$ & 37.676 & 64.8 & \\
\hline & 82.248 & $\begin{array}{l}31.0 \\
77.6\end{array}$ & & & $5 \mathrm{H}-1,40.5-40.6$ & 37.905 & & \\
\hline $\begin{array}{l}9 \mathrm{H}-5,44.8-44.9 \\
9 \mathrm{H}-6,113-112.9\end{array}$ & $\begin{array}{l}82.248 \\
84.43\end{array}$ & 1037 & & & $5 \mathrm{H}-1,57.2-57.3$ & 38.072 & 69 & \\
\hline $\begin{array}{l}9 \mathrm{H}-6,113-112.9 \\
9 \mathrm{H}-6,26.8-26.9\end{array}$ & $\begin{array}{l}84.43 \\
83.568\end{array}$ & $\begin{array}{c}103.1 \\
55\end{array}$ & & & $5 \mathrm{H}-1,71.9-72$ & 38.219 & 62.4 & \\
\hline $10 \mathrm{H}-1,119-118.7$ & $\begin{array}{l}03.508 \\
86.49\end{array}$ & 59.5 & & & $5 \mathrm{H}-1,139.6-139.7$ & 38.896 & 69.9 & \\
\hline $10 \mathrm{H}-1,37.5-37.6$ & 85.675 & 30.2 & & & $5 \mathrm{H}-2,43.7-43.8$ & 39.437 & 80.1 & \\
\hline $10 \mathrm{H}-2,144-143.8$ & 88.24 & 94 & & & $5 \mathrm{H}-2,68.9-69$ & 39.689 & 96.6 & \\
\hline
\end{tabular}


Table 2 (continued).

\begin{tabular}{|c|c|c|c|c|c|c|c|}
\hline $\begin{array}{l}\text { Core, section, } \\
\text { interval }(\mathrm{cm})\end{array}$ & $\begin{array}{l}\text { Depth } \\
\text { (mbsf) }\end{array}$ & $\begin{array}{l}\text { Strength } \\
(\mathrm{kPa})\end{array}$ & Sapropel & $\begin{array}{l}\text { Distance to fault } \\
(\mathrm{cm})\end{array}$ & $\begin{array}{l}\text { Core, section, } \\
\text { interval }(\mathrm{cm})\end{array}$ & $\begin{array}{l}\text { Depth } \\
\text { (mbsf) }\end{array}$ & $\begin{array}{l}\text { Strength } \\
(\mathrm{kPa})\end{array}$ \\
\hline $5 \mathrm{H}-2,112.8-112.9$ & 40.128 & 82.3 & & & $6 \mathrm{H}-7,45-45.1$ & 54.25 & 120 \\
\hline $5 \mathrm{H}-2,133.2-133.3$ & 40.332 & 68.8 & & & $6 \mathrm{H}-7,55-55.1$ & 54.35 & 110 \\
\hline $8 \mathrm{H}-2,13.3-13.4$ & 67.633 & 103.4 & & & $6 \mathrm{H}-7,65-65.1$ & 54.45 & 140 \\
\hline $8 \mathrm{H}-2,41-41.1$ & 67.91 & 109.4 & & & $7 \mathrm{H}-3,10-10.1$ & 57.4 & 225 \\
\hline $8 \mathrm{H}-2,69.8-69.9$ & 68.198 & 117.7 & & & $7 \mathrm{H}-3,20-20.1$ & 57.5 & 200 \\
\hline $8 \mathrm{H}-2,90.9-91$ & 68.409 & 131.7 & & & $7 \mathrm{H}-3,30-30.1$ & 57.6 & 175 \\
\hline $8 \mathrm{H}-2,103.8-103.9$ & 68.538 & 95.8 & & & $7 \mathrm{H}-3,40-40.1$ & 57.7 & 140 \\
\hline $8 \mathrm{H}-2,119.3-119.4$ & 68.693 & 114.6 & & & $7 \mathrm{H}-3,50-50.1$ & 57.8 & 150 \\
\hline $8 \mathrm{H}-3,8-8.1$ & 69.08 & 75.9 & & & $7 \mathrm{H}-3,60-60.1$ & 57.9 & 160 \\
\hline $8 \mathrm{H}-3,8.2-8.3$ & 69.082 & 102.6 & & & $7 \mathrm{H}-3,70-70.1$ & 58 & 145 \\
\hline $8 \mathrm{H}-3,20.6-20.7$ & 69.206 & 70 & & & $7 \mathrm{H}-3,80-80.1$ & 58.1 & 175 \\
\hline $8 \mathrm{H}-3,32.8-32.9$ & 69.328 & 73.5 & & & $7 \mathrm{H}-3,90-90.1$ & 58.2 & 160 \\
\hline $8 \mathrm{H}-3,39.9-40$ & 69.399 & 121.9 & & & $7 \mathrm{H}-3,95-95.1$ & 58.25 & 130 \\
\hline $8 \mathrm{H}-3,45-45.1$ & 69.45 & 74.4 & & & $7 \mathrm{H}-3,100-100.1$ & 58.3 & 110 \\
\hline $8 \mathrm{H}-3,59.4-59.5$ & 69.594 & 93.7 & & & $7 \mathrm{H}-3,110-110.1$ & 58.4 & 120 \\
\hline $8 \mathrm{H}-3,68.7-68.8$ & 69.687 & 97 & & & $7 \mathrm{H}-3,120-120.1$ & 58.5 & 80 \\
\hline $8 \mathrm{H}-3,91.5-91.6$ & 69.915 & 112 & & & $7 \mathrm{H}-3,130-130.1$ & 58.6 & 160 \\
\hline $8 \mathrm{H}-3,101.2-101.3$ & 70.012 & 143.9 & & & $7 \mathrm{H}-3,145-145.1$ & 58.75 & 100 \\
\hline $8 \mathrm{H}-3,111.5-111.6$ & 70.115 & 112.1 & & & $7 \mathrm{H}-4,10-10.1$ & 58.9 & 125 \\
\hline $8 \mathrm{H}-3,119.6-119.7$ & 70.196 & 111.8 & & & $7 \mathrm{H}-4,15-15.1$ & 58.95 & 130 \\
\hline $8 \mathrm{H}-3,144.4-144.5$ & 70.444 & 108.4 & & & $7 \mathrm{H}-4,20-20.1$ & 59 & 200 \\
\hline $8 \mathrm{H}-4,17.9-18$ & 70.679 & 115.5 & & & $7 \mathrm{H}-4,25-25.1$ & 59.05 & 210 \\
\hline $8 \mathrm{H}-4,57.6-57.7$ & 71.076 & 107.8 & & & $7 \mathrm{H}-4,30-30.1$ & 59.1 & 220 \\
\hline $8 \mathrm{H}-4,107.8-107.9$ & 71.578 & 145.9 & & & $7 \mathrm{H}-4,35-35.1$ & 59.15 & 375 \\
\hline $160-964 \mathrm{~A}-$ & & & & & $7 \mathrm{H}-4,50-50.1$ & 59.3 & 130 \\
\hline 160-964A- & & & & & $7 \mathrm{H}-4,55-55.1$ & 59.35 & 220 \\
\hline & 49.479 & 325 & & & $7 \mathrm{H}-4,60-60.1$ & 59.4 & 260 \\
\hline $6 \mathrm{H}-4,20-20.1$ & 49.529 & 245 & & & $7 \mathrm{H}-4,70-70.1$ & 59.5 & 260 \\
\hline $6 \mathrm{H}-4,25-25.1$ & 49.579 & 265 & & & $7 \mathrm{H}-4,85-85.1$ & 59.65 & 280 \\
\hline $6 \mathrm{H}-4,30-30.1$ & 49.629 & 285 & + & 10 & $7 \mathrm{H}-4,100-100.1$ & 59.8 & 175 \\
\hline $6 \mathrm{H}-4,35-35.1$ & 49.679 & 185 & + & 15 & $7 \mathrm{H}-4,105-105.1$ & 59.85 & 380 \\
\hline $6 \mathrm{H}-4,40-40.1$ & 49.729 & 90 & & & $7 \mathrm{H}-4,115-115.1$ & 59.95 & 280 \\
\hline $6 \mathrm{H}-4,45-45.1$ & 49.779 & 170 & & & $7 \mathrm{H}-4,120-120.1$ & $\begin{array}{l}59.95 \\
60\end{array}$ & 230 \\
\hline $6 \mathrm{H}-4,50-50.1$ & 49.829 & 180 & & & $7 \mathrm{H}-4,125-125.1$ & 60.05 & 350 \\
\hline $6 \mathrm{H}-4,60-60.1$ & 49.929 & 160 & & & $7 \mathrm{H}-4,130-130.1$ & 60.1 & 275 \\
\hline $6 \mathrm{H}-4,70-70.1$ & 50.029 & 225 & & & $7 \mathrm{H}-4,140-140.1$ & 60.2 & 200 \\
\hline $6 \mathrm{H}-4,80-80.1$ & 50.129 & 180 & & & $6 \mathrm{H}-4,5-5.16 .65$ & 80 & \\
\hline $6 \mathrm{H}-4,90-90.1$ & 50.229 & 200 & & & $6 \mathrm{H}-4,10-10.1$ & 46.7 & 100 \\
\hline $6 \mathrm{H}-4,100-100.1$ & 50.329 & 150 & & & $6 \mathrm{H}-4,15-15.1$ & 46.75 & 110 \\
\hline $6 \mathrm{H}-4,110-110.1$ & 50.429 & 180 & & & $6 \mathrm{H}-4,20-20.1$ & 46.8 & 175 \\
\hline $6 \mathrm{H}-4,115-115.1$ & 50.479 & 150 & & & $6 \mathrm{H}-4,30-30.1$ & $\begin{array}{l}40.0 \\
46.9\end{array}$ & 80 \\
\hline $6 \mathrm{H}-4,125-125.1$ & 50.579 & 110 & & & $6 \mathrm{H}-4,40-40.1$ & 47 & 120 \\
\hline $6 \mathrm{H}-4,135-135.1$ & 50.679 & 110 & & & $6 \mathrm{H}-4,45-45.1$ & 47.05 & 135 \\
\hline $6 \mathrm{H}-4,145-145.1$ & 50.779 & 100 & & & $6 \mathrm{H}-4,55-55.1$ & 47.15 & 85 \\
\hline $6 \mathrm{H}-4,150-150.1$ & 50.829 & 80 & & & $6 \mathrm{H}-4,65-65.1$ & 47.25 & 175 \\
\hline $6 \mathrm{H}-5,5-5.1$ & 50.55 & 90 & & & $6 \mathrm{H}-4,75-75.1$ & 47.35 & 120 \\
\hline $6 \mathrm{H}-5,10-10.1$ & 50.6 & 175 & & & $6 \mathrm{H}-4,90-90.1$ & 47.5 & 130 \\
\hline $6 \mathrm{H}-5,15-15.1$ & 50.65 & 150 & + & 20 & $\begin{array}{l}6 \mathrm{H}-4,90-90.1 \\
6 \mathrm{H}-4,110-110.1\end{array}$ & 47.7 & 120 \\
\hline $6 \mathrm{H}-5,20-20.1$ & 50.7 & 150 & + & 15 & $6 \mathrm{H}-4,125-125.1$ & 47.85 & 40 \\
\hline $6 \mathrm{H}-5,25-25.1$ & 50.75 & 100 & & & $6 \mathrm{H}-4,135-135.1$ & 47.95 & 60 \\
\hline $6 \mathrm{H}-5,30-30.1$ & 50.8 & 175 & & & $6 \mathrm{H}-4,140-140.1$ & 48 & 60 \\
\hline $6 \mathrm{H}-5,35-35.1$ & 50.85 & 150 & & & $6 \mathrm{H}-5,5-5.1$ & 48.15 & 125 \\
\hline $6 \mathrm{H}-5,40-40.1$ & 50.9 & 125 & & & $6 \mathrm{H}-5,15-15.1$ & 48.25 & 100 \\
\hline $6 \mathrm{H}-5,45-45.1$ & 50.95 & 125 & & & $6 \mathrm{H}-5,30-30.1$ & 48.4 & 125 \\
\hline $6 \mathrm{H}-5,50-50.1$ & 51 & 100 & & & $6 \mathrm{H}-5,40-40.1$ & $\begin{array}{l}40.4 \\
48.5\end{array}$ & 75 \\
\hline $6 \mathrm{H}-5,55-55.1$ & 51.05 & 120 & & & $6 \mathrm{H}-5,60-60.1$ & 48.7 & 125 \\
\hline $6 \mathrm{H}-5,60-60.1$ & 51.1 & 140 & & & $6 \mathrm{H}-5,65-65.1$ & 48.75 & 150 \\
\hline $6 \mathrm{H}-5,65-65.1$ & 51.15 & 80 & & & $6 \mathrm{H}-5,75-75.1$ & 48.85 & 100 \\
\hline $6 \mathrm{H}-5,70-70.1$ & 51.2 & 90 & & & $6 \mathrm{H}-5,80-80.1$ & $\begin{array}{l}40.03 \\
48.9\end{array}$ & 50 \\
\hline $6 \mathrm{H}-5,75-75.1$ & 51.25 & 120 & & & $6 \mathrm{H}-5,90-90.1$ & 49 & 80 \\
\hline $6 \mathrm{H}-5,80-80.1$ & 51.3 & 110 & & & $6 \mathrm{H}-5,100-100.1$ & 49.1 & 75 \\
\hline $6 \mathrm{H}-5,85-85.1$ & 51.35 & 125 & & & $6 \mathrm{H}-5,110-110.1$ & $\begin{array}{l}49.1 \\
49.2\end{array}$ & 100 \\
\hline $6 \mathrm{H}-5,90-90.1$ & 51.4 & 125 & & & $6 \mathrm{H}-5,120-120.1$ & 49.3 & $\begin{array}{r}100 \\
50\end{array}$ \\
\hline $6 \mathrm{H}-5,95-95.1$ & 51.45 & 90 & & & $6 \mathrm{H}-5,130-130.1$ & 49.4 & 75 \\
\hline $6 \mathrm{H}-5,100-100.1$ & 51.5 & 130 & & & $6 \mathrm{H}-5,140-140.1$ & 49.5 & 50 \\
\hline $6 \mathrm{H}-5,105-105.1$ & 51.55 & 150 & & & $\begin{array}{l}6 \mathrm{H}-5,140-140.1 \\
6 \mathrm{H}-7,5-5.1\end{array}$ & $\begin{array}{l}49.3 \\
51.15\end{array}$ & $\begin{array}{l}50 \\
80\end{array}$ \\
\hline $6 \mathrm{H}-5,110-110.1$ & 51.6 & 125 & & & $6 \mathrm{H}-7,10-10.1$ & 51.2 & 100 \\
\hline $6 \mathrm{H}-5,115-115.1$ & 51.65 & 150 & & & $6 \mathrm{H}-7,15-15.1$ & 51.25 & 110 \\
\hline $6 \mathrm{H}-5,120-120.1$ & 51.7 & 160 & & & $\begin{array}{l}0 \mathrm{H}-1,15-15.1 \\
6 \mathrm{H}-7,20-20.1\end{array}$ & $\begin{array}{l}51.25 \\
51.3\end{array}$ & $\begin{array}{r}110 \\
90\end{array}$ \\
\hline $6 \mathrm{H}-5,125-125.1$ & 51.75 & 130 & & & $6 \mathrm{H}-7,25-25.1$ & 51.35 & 90 \\
\hline $6 \mathrm{H}-5,130-130.1$ & 51.8 & 110 & & & $6 \mathrm{H}-7,40-40.1$ & 51.5 & 120 \\
\hline $6 \mathrm{H}-5,135-135.1$ & 51.85 & 135 & & & $6 \mathrm{H}-7,50-50.1$ & 51.6 & $\begin{array}{r}120 \\
70\end{array}$ \\
\hline $6 \mathrm{H}-5,140-140.1$ & 51.9 & 120 & & & $6 \mathrm{H}-7,60-60.1$ & 51.7 & 90 \\
\hline $6 \mathrm{H}-6,10-10.1$ & 52.4 & 100 & & & $7 \mathrm{H}-1,5-5.1$ & 54.35 & 130 \\
\hline $6 \mathrm{H}-6,20-20.1$ & 52.5 & 115 & & & $7 \mathrm{H}-1,15-15.1$ & $\begin{array}{l}54.53 \\
54.45\end{array}$ & $\begin{array}{r}150 \\
80\end{array}$ \\
\hline $6 \mathrm{H}-6,30-30.1$ & 52.6 & 110 & & & $7 \mathrm{H}-1,25-25.1$ & 54.55 & 80 \\
\hline $6 \mathrm{H}-6,45-45.1$ & 52.75 & 120 & & & $7 \mathrm{H}-1,35-35.1$ & 54.65 & 225 \\
\hline $6 \mathrm{H}-6,60-60.1$ & 52.9 & 220 & & & $\begin{array}{l}7 \mathrm{H}-1,53-53.1 \\
7 \mathrm{H}-1,50-50.1\end{array}$ & $\begin{array}{l}54.05 \\
54.8\end{array}$ & 210 \\
\hline $6 \mathrm{H}-6,70-70.1$ & 53 & 100 & & & $\begin{array}{l}7 \mathrm{H}-1,50-50.1 \\
7 \mathrm{H}-1,60-60.1\end{array}$ & $\begin{array}{l}54.8 \\
54.9\end{array}$ & $\begin{array}{l}210 \\
125\end{array}$ \\
\hline $6 \mathrm{H}-6,75-75.1$ & 53.05 & 130 & & & $7 \mathrm{H}-1,70-70.1$ & 55 & 110 \\
\hline $6 \mathrm{H}-6,85-85.1$ & 53.15 & 230 & + & 50 & $7 \mathrm{H}-1,80-80.1$ & 55.1 & 165 \\
\hline $6 \mathrm{H}-6,95-95.1$ & 53.25 & 125 & + & 40 & $7 \mathrm{H}-1,90-90.1$ & 55.2 & 120 \\
\hline $6 \mathrm{H}-6,105-105.1$ & 53.35 & 85 & & & $7 \mathrm{H}-1,100-100.1$ & 55.3 & 240 \\
\hline $6 \mathrm{H}-6,115-115.1$ & 53.45 & 175 & & & $7 \mathrm{H}-1,110-110.1$ & 55.4 & 80 \\
\hline $6 \mathrm{H}-6,125-125.1$ & 53.55 & 180 & & & $7 \mathrm{H}-1,120-120.1$ & 55.5 & 95 \\
\hline $6 \mathrm{H}-6,135-135.1$ & 53.65 & 125 & & & $\begin{array}{l}7 \mathrm{H}-1,120-120.1 \\
7 \mathrm{H}-1,125-125.1\end{array}$ & $\begin{array}{l}5.5 \\
55.55\end{array}$ & $\begin{array}{l}93 \\
65\end{array}$ \\
\hline $6 \mathrm{H}-6,145-145.1$ & 53.75 & 115 & & & $7 \mathrm{H}-1,130-130.1$ & 55.6 & 115 \\
\hline $6 \mathrm{H}-7,10-10.1$ & 53.9 & 130 & & & $7 \mathrm{H}-1,135-135.1$ & 55.65 & 80 \\
\hline $6 \mathrm{H}-7,20-20.1$ & 54 & 160 & + & 0 & & 55.75 & 110 \\
\hline $6 \mathrm{H}-7,30-30.1$ & 54.1 & 125 & & & $7 \mathrm{H}-1,145-145.1$ & 55.15 & 110 \\
\hline
\end{tabular}


Table 2 (continued).

\begin{tabular}{|c|c|c|c|c|c|c|c|c|}
\hline $\begin{array}{l}\text { Core, section, } \\
\text { interval }(\mathrm{cm})\end{array}$ & $\begin{array}{l}\text { Depth } \\
\text { (mbsf) }\end{array}$ & $\begin{array}{l}\text { Strength } \\
(\mathrm{kPa})\end{array}$ & Sapropel & $\begin{array}{l}\text { Distance to fault } \\
(\mathrm{cm})\end{array}$ & $\begin{array}{l}\text { Core, section, } \\
\text { interval }(\mathrm{cm})\end{array}$ & $\begin{array}{l}\text { Depth } \\
\text { (mbsf) }\end{array}$ & $\begin{array}{l}\text { Strength } \\
(\mathrm{kPa})\end{array}$ & Sapropel \\
\hline 160-965A- & & & & & $6 \mathrm{H}-3,20-20.1$ & 50.5 & 90 & \\
\hline $2 \mathrm{H}-7,5-5.1$ & 10.7 & 100 & & & $6 \mathrm{H}-3,30-30.1$ & 50.6 & 115 & + \\
\hline $2 \mathrm{H}-7,10-10.1$ & 10.75 & 110 & & & $6 \mathrm{H}-3,35-35.1$ & 50.65 & 160 & + \\
\hline $2 \mathrm{H}-7,15-15.1$ & 10.8 & 120 & & & $6 \mathrm{H}-3,40-40.1$ & 50.7 & 130 & + \\
\hline $2 \mathrm{H}-7,20-20.1$ & 10.85 & 175 & & & $6 \mathrm{H}-3,45-45.1$ & 50.75 & 90 & \\
\hline $2 \mathrm{H}-7,25-25.1$ & 10.9 & 225 & & & $6 \mathrm{H}-3,55-55.1$ & 50.85 & 90 & \\
\hline $2 \mathrm{H}-7,30-30.1$ & 10.95 & 165 & & & $6 \mathrm{H}-3,65-65.1$ & 50.95 & 100 & \\
\hline $2 \mathrm{H}-7,35-35.1$ & 11 & 145 & & & $6 \mathrm{H}-3,75-75.1$ & 51.05 & 135 & + \\
\hline $2 \mathrm{H}-7,40-40.1$ & 11.05 & 40 & & & $6 \mathrm{H}-3,85-85.1$ & 51.15 & 80 & \\
\hline $2 \mathrm{H}-7,45-45.1$ & 11.1 & 90 & & & $6 \mathrm{H}-3,95-95.1$ & 51.25 & 75 & \\
\hline $2 \mathrm{H}-7,50-50.1$ & 11.15 & 115 & + & 50 & $6 \mathrm{H}-3,105-105.1$ & 51.35 & 85 & \\
\hline $2 \mathrm{H}-7,55-55.1$ & 11.2 & 215 & + & 45 & $6 \mathrm{H}-3,115-115.1$ & 51.45 & 75 & \\
\hline $2 \mathrm{H}-7,60-60.1$ & 11.25 & 210 & & & $6 \mathrm{H}-3,125-125.1$ & 51.55 & 100 & \\
\hline $2 \mathrm{H}-7,65-65.1$ & 11.3 & 135 & & & $6 \mathrm{H}-3,135-135.1$ & 51.65 & 95 & \\
\hline $3 \mathrm{H}-1,5-5.1$ & 10.35 & 30 & & & $6 \mathrm{H}-3,145-145.1$ & 51.75 & 125 & \\
\hline $3 \mathrm{H}-1,10-10.1$ & 10.4 & 45 & & & $6 \mathrm{H}-4,10-10.1$ & 51.9 & 115 & \\
\hline $3 \mathrm{H}-1,15-15.1$ & 10.45 & 50 & + & 0 & $6 \mathrm{H}-4,20-20.1$ & 52 & 110 & \\
\hline $3 \mathrm{H}-1,20-20.1$ & 10.5 & 45 & & & $6 \mathrm{H}-4,30-30.1$ & 52.1 & 155 & \\
\hline $3 \mathrm{H}-1,25-25.1$ & 10.55 & 50 & & & $6 \mathrm{H}-4,35-35.1$ & 52.15 & 230 & + \\
\hline $3 \mathrm{H}-1,30-30.1$ & 10.6 & 70 & & & $6 \mathrm{H}-4,40-40.1$ & 52.2 & 160 & + \\
\hline $3 \mathrm{H}-1,35-35.1$ & 10.65 & 110 & & & $6 \mathrm{H}-4,45-45.1$ & 52.25 & 110 & \\
\hline $3 \mathrm{H}-1,40-40.1$ & 10.7 & 60 & & & $6 \mathrm{H}-4,55-55.1$ & 52.35 & 100 & \\
\hline $3 \mathrm{H}-1,45-45.1$ & 10.75 & 65 & & & $6 \mathrm{H}-4,65-65.1$ & 52.45 & 110 & \\
\hline $3 \mathrm{H}-1,50-50.1$ & 10.8 & 75 & & & $6 \mathrm{H}-4,70-70.1$ & 52.5 & 85 & \\
\hline $3 \mathrm{H}-1,55-55.1$ & 10.85 & 80 & & & $6 \mathrm{H}-4,75-75.1$ & 52.55 & 110 & \\
\hline $3 \mathrm{H}-1,60-60.1$ & 10.9 & 85 & & & $6 \mathrm{H}-4,80-80.1$ & 52.6 & 120 & \\
\hline $3 \mathrm{H}-2,5-5.1$ & 11.85 & 65 & & & $6 \mathrm{H}-4,85-85.1$ & 52.65 & 145 & + \\
\hline $3 \mathrm{H}-2,10-10.1$ & 11.9 & 85 & & & $6 \mathrm{H}-4,90-90.1$ & 52.7 & 105 & \\
\hline $3 \mathrm{H}-2,15-15.1$ & 11.95 & 65 & & & $6 \mathrm{H}-4,100-100.1$ & 52.8 & 120 & \\
\hline $3 \mathrm{H}-2,20-20.1$ & 12 & 110 & & & $6 \mathrm{H}-4,110-110.1$ & 52.9 & 130 & \\
\hline $3 \mathrm{H}-2,25-25.1$ & 12.05 & 75 & & & $6 \mathrm{H}-4,120-120.1$ & 53 & 120 & \\
\hline $3 \mathrm{H}-2,30-30.1$ & 12.1 & 65 & & & $6 \mathrm{H}-4,125-125.1$ & 53.05 & 185 & + \\
\hline $3 \mathrm{H}-2,35-35.1$ & 12.15 & 60 & & & $6 \mathrm{H}-4,130-130.1$ & 53.1 & 135 & + \\
\hline $3 \mathrm{H}-2,40-40.1$ & 12.2 & 40 & & & $6 \mathrm{H}-4,135-135.1$ & 53.15 & 135 & + \\
\hline $3 \mathrm{H}-2,45-45.1$ & 12.25 & 75 & & & $6 \mathrm{H}-4,140-140.1$ & 53.2 & 180 & \\
\hline $3 \mathrm{H}-2,50-50.1$ & 12.3 & 100 & & & $6 \mathrm{H}-4,145-145.1$ & 53.25 & 95 & \\
\hline $3 \mathrm{H}-2,0-60.1$ & 12.4 & 95 & & & $6 \mathrm{H}-4,150-150.1$ & 53.3 & 125 & \\
\hline $3 \mathrm{H}-2,65-5.1$ & 12.45 & 80 & & & $6 \mathrm{H}-5,50-50.1$ & 53.8 & 140 & \\
\hline $3 \mathrm{H}-2,70-0.1$ & 12.5 & 85 & & & $6 \mathrm{H}-5,60-60.1$ & 53.9 & 150 & \\
\hline $3 \mathrm{H}-3,5-5.1$ & 13.35 & 60 & & & $6 \mathrm{H}-5,70-70.1$ & 54 & 130 & \\
\hline $3 \mathrm{H}-3,10-10.1$ & 13.4 & 85 & & & $6 \mathrm{H}-5,80-80.1$ & 54.1 & 130 & \\
\hline $3 \mathrm{H}-3,15-15.1$ & 13.45 & 95 & & & $6 \mathrm{H}-5,90-90.1$ & 54.2 & 145 & \\
\hline $3 \mathrm{H}-3,20-20.1$ & 13.5 & 90 & & & $6 \mathrm{H}-5,100-100.1$ & 54.3 & 130 & \\
\hline $3 \mathrm{H}-3,25-25.1$ & 13.55 & 85 & & & $6 \mathrm{H}-5,110-110.1$ & 54.4 & 130 & \\
\hline $3 \mathrm{H}-3,30-30.1$ & 13.6 & 80 & & & $6 \mathrm{H}-5,125-125.1$ & 54.55 & 160 & \\
\hline $3 \mathrm{H}-3,35-5.1$ & 13.65 & 95 & & & $6 \mathrm{H}-5,130-130.1$ & 54.6 & 160 & \\
\hline $3 \mathrm{H}-3,40-40.1$ & 13.7 & 80 & & & $6 \mathrm{H}-5,135-135.1$ & 54.65 & 125 & \\
\hline $3 \mathrm{H}-3,45-45.1$ & 13.75 & 80 & & & $6 \mathrm{H}-5,140-140.1$ & 54.7 & 150 & \\
\hline $3 \mathrm{H}-3,50-50.1$ & 13.8 & 80 & & & $6 \mathrm{H}-5,145-145.1$ & 54.75 & 130 & \\
\hline $3 \mathrm{H}-3,55-5.1$ & 13.85 & 75 & & & $8 \mathrm{H}-1,5-5.1$ & 66.35 & 130 & \\
\hline $3 \mathrm{H}-3,60-60.1$ & 13.9 & 50 & & & $8 \mathrm{H}-1,10-10.1$ & 66.4 & 160 & \\
\hline $3 \mathrm{H}-3,65-5.1$ & 13.95 & 65 & & & $8 \mathrm{H}-1,15-15.1$ & 66.45 & 140 & \\
\hline $3 \mathrm{H}-3,70-70.1$ & 14 & 70 & & & $8 \mathrm{H}-1,20-20.1$ & 66.5 & 150 & + \\
\hline $6 \mathrm{H}-2,30-30.1$ & 43.3 & 110 & & & $8 \mathrm{H}-1,25-25.1$ & 66.55 & 140 & + \\
\hline $6 \mathrm{H}-2,40-40.1$ & 43.4 & 110 & & & $8 \mathrm{H}-1,30-30.1$ & 66.6 & 145 & + \\
\hline $6 \mathrm{H}-2,50-50.1$ & 43.5 & 125 & B & 50 & $8 \mathrm{H}-1,35-35.1$ & 66.65 & 120 & + \\
\hline & & & & & $8 \mathrm{H}-1,40-40.1$ & 66.7 & 110 & \\
\hline 160-966D- & & & & & $8 \mathrm{H}-1,45-45.1$ & 66.75 & 135 & \\
\hline $6 \mathrm{H}-2,60-60.1$ & 43.6 & 100 & & & $8 \mathrm{H}-1,50-50.1$ & 66.8 & 135 & \\
\hline $6 \mathrm{H}-2,70-70.1$ & 43.7 & 80 & & & $8 \mathrm{H}-1,55-55.1$ & 66.85 & 130 & \\
\hline $6 \mathrm{H}-2,75-75.1$ & 43.75 & 80 & & & $8 \mathrm{H}-1,60-60.1$ & 66.9 & 130 & \\
\hline $6 \mathrm{H}-2,80-80.1$ & 43.8 & 120 & & & $8 \mathrm{H}-1,65-65.1$ & 66.95 & 130 & \\
\hline $6 \mathrm{H}-2,85-85.1$ & 43.85 & 100 & & & $8 \mathrm{H}-1,70-70.1$ & 67 & 140 & \\
\hline $6 \mathrm{H}-2,95-95.1$ & 43.95 & 110 & B & 10 & $8 \mathrm{H}-1,75-75.1$ & 67.05 & 140 & \\
\hline $6 \mathrm{H}-2,130-130.1$ & 44.3 & 80 & & & $8 \mathrm{H}-1,80-80.1$ & 67.1 & 110 & \\
\hline $6 \mathrm{H}-2,140-140.1$ & 44.4 & 90 & & & $8 \mathrm{H}-1,85-85.1$ & 67.15 & 145 & + \\
\hline $6 \mathrm{H}-2,145-145.1$ & 44.45 & 80 & B & 0 & $8 \mathrm{H}-1,90-90.1$ & 67.2 & 200 & + \\
\hline $6 \mathrm{H}-3,10-10.1$ & 44.6 & 125 & & & $8 \mathrm{H}-1,95-95.1$ & 67.25 & 260 & + \\
\hline $6 \mathrm{H}-3,20-20.1$ & 44.7 & 25 & & & $8 \mathrm{H}-1,100-100.1$ & 67.3 & 130 & \\
\hline $6 \mathrm{H}-3,30-30.1$ & 44.8 & 100 & B & 5 & $8 \mathrm{H}-1,105-105.1$ & 67.35 & 165 & \\
\hline $6 \mathrm{H}-3,40-40.1$ & 44.9 & 120 & & & $8 \mathrm{H}-1,110-110.1$ & 67.4 & 160 & \\
\hline $6 \mathrm{H}-3,50-50.1$ & 45 & 80 & & & $8 \mathrm{H}-1,115-115.1$ & 67.45 & 150 & \\
\hline $6 \mathrm{H}-3,60-60.1$ & 45.1 & 90 & B & 25 & $8 \mathrm{H}-1,120-120.1$ & 67.5 & 170 & \\
\hline $6 \mathrm{H}-3,75-75.1$ & 45.25 & 160 & & & $8 \mathrm{H}-1,125-125.1$ & 67.55 & 160 & \\
\hline $6 \mathrm{H}-3,85-85.1$ & 45.35 & 160 & & & $8 \mathrm{H}-1,130-130.1$ & 67.6 & 150 & \\
\hline $6 \mathrm{H}-3,95-95.1$ & 45.45 & 100 & & & $8 \mathrm{H}-1,135-135.1$ & 67.65 & 165 & \\
\hline $6 \mathrm{H}-3,105-105.1$ & 45.55 & 150 & & & $8 \mathrm{H}-1,140-140.1$ & 67.7 & 170 & \\
\hline $6 \mathrm{H}-3,115-115.1$ & $\begin{array}{l}45.65 \\
458\end{array}$ & $\begin{array}{r}70 \\
140\end{array}$ & & & $8 \mathrm{H}-1,145-145.1$ & 67.75 & 200 & \\
\hline $\begin{array}{l}6 \mathrm{H}-3,130-130.1 \\
6 \mathrm{H}-3,140-140.1\end{array}$ & $\begin{array}{l}45.8 \\
45.9\end{array}$ & $\begin{array}{l}140 \\
125\end{array}$ & B & 0 & $160-967 C-$ & & & \\
\hline $6 \mathrm{H}-3,150-150.1$ & 46 & 125 & & & $6 \mathrm{H}-4,5-5.1$ & 52.25 & 155 & + \\
\hline $7 \mathrm{H}-3,90-90.1$ & 54.88 & 175 & B & 240 & $6 \mathrm{H}-4,10-10.1$ & 52.3 & 110 & + \\
\hline $7 \mathrm{H}-3,100-100.1$ & 54.98 & 170 & & & $6 \mathrm{H}-4,20-20.1$ & 52.35 & 80 & \\
\hline $7 \mathrm{H}-3,110-110.1$ & 55.08 & 175 & & & $6 \mathrm{H}-4,30-30.1$ & 52.4 & 150 & \\
\hline $7 \mathrm{H}-3,130-130.1$ & 55.28 & 165 & & & $6 \mathrm{H}-4,40-40.1$ & 52.45 & 110 & \\
\hline $7 \mathrm{H}-3,135-135.1$ & 55.33 & 190 & & & $6 \mathrm{H}-4,50-50.1$ & 52.5 & 175 & + \\
\hline $7 \mathrm{H}-3,145-145.1$ & 55.43 & 180 & B & 0 & $6 \mathrm{H}-4,55-55.1$ & 52.55 & 110 & \\
\hline & & & & & $6 \mathrm{H}-4,60-60.1$ & 52.6 & 140 & \\
\hline 160-96/A- & & & & & $6 \mathrm{H}-4,65-65.1$ & 52.65 & 150 & \\
\hline $6 \mathrm{H}-3,10-10.1$ & 50.4 & 110 & & & $6 \mathrm{H}-4,70-70.1$ & 52.7 & 160 & \\
\hline
\end{tabular}


Table 2 (continued).

\begin{tabular}{|c|c|c|c|c|c|c|c|}
\hline $\begin{array}{l}\text { Core, section, } \\
\text { interval }(\mathrm{cm})\end{array}$ & $\begin{array}{l}\text { Depth } \\
\text { (mbsf) }\end{array}$ & $\begin{array}{l}\text { Strength } \\
(\mathrm{kPa})\end{array}$ & Sapropel & $\begin{array}{l}\text { Distance to fault } \\
(\mathrm{cm})\end{array}$ & $\begin{array}{l}\text { Core, section, } \\
\text { interval }(\mathrm{cm})\end{array}$ & $\begin{array}{l}\text { Depth } \\
\text { (mbsf) }\end{array}$ & $\begin{array}{l}\text { Strength } \\
(\mathrm{kPa})\end{array}$ \\
\hline $6 \mathrm{H}-4,75-75.1$ & 52.75 & 165 & & & $4 \mathrm{H}-4,130-130.1$ & 33.8 & 210 \\
\hline $6 \mathrm{H}-4,80-80.1$ & 52.8 & 155 & & & $4 \mathrm{H}-4,135-135.1$ & 33.85 & 120 \\
\hline $6 \mathrm{H}-4,85-85.1$ & 52.85 & 140 & & & $4 \mathrm{H}-4,140-140.1$ & 33.9 & 190 \\
\hline $6 \mathrm{H}-4,90-90.1$ & 52.9 & 180 & & & $4 \mathrm{H}-5,10-10.1$ & 34.1 & 210 \\
\hline $6 \mathrm{H}-4,95-95.1$ & 52.95 & 150 & & & $4 \mathrm{H}-5,15-15.1$ & 34.15 & 180 \\
\hline $6 \mathrm{H}-4,100-100.1$ & 53 & 215 & & & $4 \mathrm{H}-5,20-20.1$ & 34.2 & 190 \\
\hline $6 \mathrm{H}-4,105-105.1$ & 53.05 & 225 & & & $4 \mathrm{H}-5,30-30.1$ & 34.3 & 175 \\
\hline $6 \mathrm{H}-4,110-110.1$ & 53.1 & 170 & & & $4 \mathrm{H}-5,40-40.1$ & 34.4 & 200 \\
\hline $6 \mathrm{H}-4,115-115.1$ & 53.15 & 150 & & & $4 \mathrm{H}-5,50-50.1$ & 34.5 & 180 \\
\hline $6 \mathrm{H}-4,120-120.1$ & 53.2 & 180 & & & $4 \mathrm{H}-5,60-60.1$ & 34.6 & 280 \\
\hline $6 \mathrm{H}-4,125-125.1$ & 53.25 & 155 & & & $4 \mathrm{H}-5,70-70.1$ & 34.7 & 220 \\
\hline $6 \mathrm{H}-4,130-130.1$ & 53.3 & 225 & + & 0 & $4 \mathrm{H}-5,80-80.1$ & 34.8 & 200 \\
\hline $6 \mathrm{H}-4,135-135.1$ & 53.35 & 180 & & & $4 \mathrm{H}-5,95-95.1$ & 34.95 & 140 \\
\hline $6 \mathrm{H}-4,140-140.1$ & 53.4 & 150 & & & $4 \mathrm{H}-5,110-110.1$ & 35.1 & 180 \\
\hline $6 \mathrm{H}-4,145-145.1$ & 53.45 & 125 & & & $4 \mathrm{H}-5,120-120.1$ & 35.2 & 190 \\
\hline $6 \mathrm{H}-4,150-150.1$ & 53.5 & 145 & & & $4 \mathrm{H}-5,125-125.1$ & 35.25 & 175 \\
\hline $6 \mathrm{H}-5,5-5.1$ & 54.05 & 140 & + & 0 & $4 \mathrm{H}-5,130-130.1$ & 35.3 & 160 \\
\hline $6 \mathrm{H}-5,10-10.1$ & 54.15 & 150 & + & 0 & $4 \mathrm{H}-5,135-135.1$ & 35.35 & 125 \\
\hline $6 \mathrm{H}-5,15-15.1$ & 54.25 & 130 & & & $4 \mathrm{H}-5,145-145.1$ & 35.45 & 150 \\
\hline $6 \mathrm{H}-5,20-20.1$ & 54.35 & 130 & & & $5 \mathrm{H}-1,5-5.1$ & 37.55 & 175 \\
\hline $6 \mathrm{H}-5,25-25.1$ & 54.45 & 145 & & & $5 \mathrm{H}-1,15-15.1$ & 37.65 & 140 \\
\hline $6 \mathrm{H}-5,30-30.1$ & 54.55 & 130 & & & $5 \mathrm{H}-1,25-25.1$ & 37.75 & 130 \\
\hline $6 \mathrm{H}-5,35-35.1$ & 54.65 & 130 & & & $5 \mathrm{H}-1,35-35.1$ & 37.85 & 120 \\
\hline $6 \mathrm{H}-5,40-40.1$ & 54.8 & 160 & & & $5 \mathrm{H}-1,45-45.1$ & 37.95 & 175 \\
\hline $6 \mathrm{H}-5,45-45.1$ & 54.85 & 160 & & & $5 \mathrm{H}-1,55-55.1$ & 38.05 & 120 \\
\hline $6 \mathrm{H}-5,50-50.1$ & 54.9 & 125 & & & $5 \mathrm{H}-1,65-65.1$ & 38.15 & 120 \\
\hline $6 \mathrm{H}-5,55-55.1$ & 54.95 & 150 & & & $5 \mathrm{H}-1,75-75.1$ & 38.25 & 125 \\
\hline $6 \mathrm{H}-5,60-60.1$ & 55 & 130 & & & $5 \mathrm{H}-1,85-85.1$ & 38.35 & 140 \\
\hline & & & & & $5 \mathrm{H}-1,95-95.1$ & 38.45 & 140 \\
\hline 160-968A- & & & & & $5 \mathrm{H}-1,105-105.1$ & 38.55 & 160 \\
\hline $4 \mathrm{H}-1,10-10.1$ & 28.1 & 130 & & & $5 \mathrm{H}-1,115-115.1$ & 152.6 & 120 \\
\hline $4 \mathrm{H}-1,25-25.1$ & 28.25 & 125 & & & $5 \mathrm{H}-1,125-125.1$ & 162.6 & 120 \\
\hline $4 \mathrm{H}-1,40-40.1$ & 28.4 & 125 & & & $5 \mathrm{H}-1,135-135.1$ & 172.6 & 125 \\
\hline $4 \mathrm{H}-1,50-50.1$ & 28.5 & 125 & & & $5 \mathrm{H}-1,145-145.1$ & 182.6 & 125 \\
\hline $4 \mathrm{H}-1,60-60.1$ & 28.6 & 165 & & & $5 \mathrm{H}-2,5-5.1$ & $\begin{array}{l}102.0 \\
39.05\end{array}$ & 130 \\
\hline $4 \mathrm{H}-1,70-70.1$ & 28.7 & 150 & & & $5 \mathrm{H}-2,15-15.1$ & 39.15 & 140 \\
\hline $4 \mathrm{H}-1,75-75.1$ & 28.75 & 145 & & & $5 \mathrm{H}-2,25-25.1$ & 39.25 & 145 \\
\hline $4 \mathrm{H}-1,85-85.1$ & 28.85 & 80 & & & $5 \mathrm{H}-2,35-35.1$ & 39.35 & 160 \\
\hline $4 \mathrm{H}-1,90-90.1$ & 28.9 & 120 & + & 10 & $5 \mathrm{H}-2,45-45.1$ & 39.45 & 170 \\
\hline $4 \mathrm{H}-1,100-100.1$ & 29 & 130 & & & $5 \mathrm{H}-2,55-55.1$ & 39.55 & 175 \\
\hline $4 \mathrm{H}-1,105-105.1$ & 29.05 & 120 & & & $5 \mathrm{H}-2,60-60.1$ & 39.6 & 175 \\
\hline $4 \mathrm{H}-1,110-110.1$ & 29.1 & 110 & & & $5 \mathrm{H}-2,70-70.1$ & 39.7 & 170 \\
\hline $4 \mathrm{H}-1,120-120.1$ & 29.2 & 115 & & & $5 \mathrm{H}-2,80-80.1$ & 39.8 & 160 \\
\hline $4 \mathrm{H}-1,125-125.1$ & 29.25 & 160 & & & $5 \mathrm{H}-2,90-90.1$ & 39.9 & 130 \\
\hline $4 \mathrm{H}-1,135-135.1$ & 29.35 & 140 & & & $5 \mathrm{H}-2,100-100.1$ & 40 & 185 \\
\hline $4 \mathrm{H}-1,145-145.1$ & 29.45 & 105 & & & $5 \mathrm{H}-2,110-110.1$ & $\begin{array}{l}40 \\
40.1\end{array}$ & 165 \\
\hline $4 \mathrm{H}-2,5-5.1$ & 29.55 & 135 & & & $5 \mathrm{H}-2,120-120.1$ & $\begin{array}{l}40.1 \\
40.2\end{array}$ & $\begin{array}{l}103 \\
190\end{array}$ \\
\hline $4 \mathrm{H}-2,15-15.1$ & 29.65 & 130 & & & $5 \mathrm{H}-2,125-125.1$ & 40.25 & 150 \\
\hline $4 \mathrm{H}-2,25-25.1$ & 29.75 & 120 & & & $5 \mathrm{H}-2,135-135.1$ & 40.35 & 130 \\
\hline $4 \mathrm{H}-2,35-35.1$ & 29.85 & 130 & & & $\begin{array}{l}5 \mathrm{H}-2,135-135.1 \\
5 \mathrm{H}-2,145-145.1\end{array}$ & 40.45 & 130 \\
\hline $4 \mathrm{H}-2,45-45.1$ & 29.95 & 125 & & & $8 \mathrm{H}-1,5-5.1$ & 66.05 & 155 \\
\hline $4 \mathrm{H}-2,55-55.1$ & 30.05 & 135 & & & $8 \mathrm{H}-1,10-10.1$ & 66.1 & 130 \\
\hline $4 \mathrm{H}-2,65-65.1$ & 30.15 & 120 & & & $8 \mathrm{H}-1,15-15.1$ & 66.15 & 135 \\
\hline $4 \mathrm{H}-2,95-95.1$ & 30.45 & 110 & & & $8 \mathrm{H}-1,25-25.1$ & 66.25 & 155 \\
\hline $4 \mathrm{H}-2,105-105.1$ & 30.55 & 125 & & & $8 \mathrm{H}-1,35-35.1$ & 66.35 & 160 \\
\hline $4 \mathrm{H}-2,115-115.1$ & 30.65 & 120 & & & $8 \mathrm{H}-1,40-40.1$ & 66.4 & $\begin{array}{l}100 \\
175\end{array}$ \\
\hline $4 \mathrm{H}-2,125-125.1$ & 30.75 & 115 & & & $8 \mathrm{H}-1,45-45.1$ & 66.45 & 170 \\
\hline $4 \mathrm{H}-2,135-135.1$ & 30.85 & 125 & & & $8 \mathrm{H}-1,50-50.1$ & 66.5 & 170 \\
\hline $4 \mathrm{H}-2,145-145.1$ & 30.95 & 120 & & & $8 \mathrm{H}-1,65-65.1$ & 66.65 & 200 \\
\hline $4 \mathrm{H}-3,5-5.1$ & 31.05 & 120 & & & $8 \mathrm{H}-1,75-75.1$ & 66.75 & 175 \\
\hline $4 \mathrm{H}-3,15-15.1$ & 31.15 & 115 & & & $8 \mathrm{H}-1,80-80.1$ & 66.8 & 185 \\
\hline $4 \mathrm{H}-3,25-25.1$ & 31.25 & 115 & & & $\begin{array}{l}8 H-1,80-80.1 \\
8 \mathrm{H}-1,90-90.1\end{array}$ & $\begin{array}{l}00.8 \\
66.9\end{array}$ & 185 \\
\hline $4 \mathrm{H}-3,35-35.1$ & 31.35 & 130 & & & $8 \mathrm{H}-1,100-100.1$ & 67 & 270 \\
\hline $4 \mathrm{H}-3,45-45.1$ & 31.45 & 140 & & & $8 \mathrm{H}-1,105-105.1$ & 67.05 & 165 \\
\hline $4 \mathrm{H}-3,55-55.1$ & 31.55 & 140 & & & $8 \mathrm{H}-1,110-110.1$ & 67.1 & 110 \\
\hline $4 \mathrm{H}-3,65-65.1$ & 31.65 & 130 & & & $8 \mathrm{H}-1,115-115.1$ & 67.15 & 180 \\
\hline $4 \mathrm{H}-3,75-75.1$ & 31.75 & 125 & & & $8 \mathrm{H}-1,125-125.1$ & 67.25 & 225 \\
\hline $4 \mathrm{H}-3,85-85.1$ & 31.85 & 160 & & & $8 \mathrm{H}-1,135-135.1$ & 67.35 & 185 \\
\hline $4 \mathrm{H}-3,90-90.1$ & 31.9 & 160 & B & 55 & $\begin{array}{l}8 \mathrm{H}-1,135-135.1 \\
8 \mathrm{H}-1,145-145.1\end{array}$ & 67.45 & 140 \\
\hline $4 \mathrm{H}-3,100-100.1$ & 32 & 130 & & & $\begin{array}{l}8 \mathrm{H}-1,143-145.1 \\
8 \mathrm{H}-2,5-5.1\end{array}$ & 67.55 & $\begin{array}{l}140 \\
155\end{array}$ \\
\hline $4 \mathrm{H}-3,110-110.1$ & 32.1 & 190 & & & $8 \mathrm{H}-2,10-10.1$ & 67.6 & 130 \\
\hline $4 \mathrm{H}-3,120-120.1$ & 32.2 & 155 & & & $8 \mathrm{H}-2,15-15.1$ & 67.65 & 135 \\
\hline $4 \mathrm{H}-3,130-130.1$ & 32.3 & 140 & & & $8 \mathrm{H}-2,25-25.1$ & 67.75 & 155 \\
\hline $4 \mathrm{H}-3,135-135.1$ & 32.35 & 175 & & & $8 \mathrm{H}-2,35-35.1$ & 67.85 & 160 \\
\hline $4 \mathrm{H}-3,140-140.1$ & 32.4 & 155 & + & 0 & $8 \mathrm{H}-2,40-40.1$ & $\begin{array}{l}01.85 \\
67.9\end{array}$ & $\begin{array}{l}100 \\
175\end{array}$ \\
\hline $4 \mathrm{H}-3,145-145.1$ & 32.45 & 130 & + & 0 & $8 \mathrm{H}-2,45-45.1$ & 67.95 & 170 \\
\hline $4 \mathrm{H}-4,5-5.1$ & 32.55 & 110 & + & 10 & $8 \mathrm{H}-2,50-50.1$ & 68 & 170 \\
\hline $4 \mathrm{H}-4,15-15.1$ & 32.65 & 120 & & & $8 \mathrm{H}-2,65-65.1$ & 68.15 & 200 \\
\hline $4 \mathrm{H}-4,25-25.1$ & 32.75 & 140 & & & $8 \mathrm{H}-2,75-75.1$ & 68.25 & 175 \\
\hline $4 \mathrm{H}-4,35-35.1$ & 32.85 & 130 & & & $8 \mathrm{H}-2,80-80.1$ & 68.3 & 185 \\
\hline $4 \mathrm{H}-4,45-45.1$ & 32.95 & 160 & & & $8 \mathrm{H}-2,90-90.1$ & 68.4 & 185 \\
\hline $4 \mathrm{H}-4,55-55.1$ & 33.05 & 165 & & & $8 \mathrm{H}-2,100-100.1$ & 68.4 & 270 \\
\hline $4 \mathrm{H}-4,60-60.1$ & 33.1 & 55 & & & $8 \mathrm{H}-2,105-105.1$ & 68.55 & 165 \\
\hline $4 \mathrm{H}-4,70-70.1$ & 33.2 & 150 & & & $8 \mathrm{H}-2,110-110.1$ & 68.6 & 110 \\
\hline $4 \mathrm{H}-4,80-80.1$ & 33.3 & 180 & + & 5 & $8 \mathrm{H}-2,115-115.1$ & 68.65 & 180 \\
\hline $4 \mathrm{H}-4,90-90.1$ & 33.4 & 185 & & & $8 \mathrm{H}-2,125-125.1$ & 68.75 & 225 \\
\hline $4 \mathrm{H}-4,100-100.1$ & 33.5 & 165 & & & $8 \mathrm{H}-2,135-135.1$ & 68.85 & 185 \\
\hline $4 \mathrm{H}-4,110-110.1$ & 33.6 & 180 & + & 10 & $8 \mathrm{H}-2,145-145.1$ & 68.95 & 140 \\
\hline $4 \mathrm{H}-4,120-120.1$ & 33.7 & 160 & & & $8 \mathrm{H}-3,5-5.1$ & 69.05 & 180 \\
\hline $4 \mathrm{H}-4,125-125.1$ & 33.75 & 170 & & & $8 \mathrm{H}-3,5-5.1$ & 69.05 & 180 \\
\hline
\end{tabular}


Table 2 (continued).

\begin{tabular}{|c|c|c|c|c|}
\hline $\begin{array}{l}\text { Core, section, } \\
\text { interval }(\mathrm{cm})\end{array}$ & $\begin{array}{l}\text { Depth } \\
\text { (mbsf) }\end{array}$ & $\begin{array}{l}\text { Strength } \\
(\mathrm{kPa})\end{array}$ & Sapropel & $\begin{array}{l}\text { Distance to fault } \\
(\mathrm{cm})\end{array}$ \\
\hline $8 \mathrm{H}-3,15-15.1$ & 69.15 & 250 & & \\
\hline $8 \mathrm{H}-3,25-25.1$ & 69.25 & 260 & & \\
\hline $8 \mathrm{H}-3,35-35.1$ & 69.35 & 250 & & \\
\hline $8 \mathrm{H}-3,45-45.1$ & 69.45 & 260 & & \\
\hline $8 \mathrm{H}-3,55-55.1$ & 69.55 & 225 & & \\
\hline $8 \mathrm{H}-3,65-65.1$ & 69.65 & 240 & & \\
\hline $8 \mathrm{H}-3,75-75.1$ & 69.75 & 290 & & \\
\hline $8 \mathrm{H}-3,85-85.1$ & 69.85 & 380 & + & 40 \\
\hline $8 \mathrm{H}-3,95-95.1$ & 69.95 & 390 & + & 45 \\
\hline $8 \mathrm{H}-3,100-100.1$ & 70 & 190 & + & 50 \\
\hline $8 \mathrm{H}-3,110-110.1$ & 70.1 & 270 & + & 60 \\
\hline $8 \mathrm{H}-3,120-120.1$ & 70.2 & 225 & + & 70 \\
\hline $8 \mathrm{H}-3,130-130.1$ & 70.3 & 210 & + & 80 \\
\hline $8 \mathrm{H}-3,140-140.1$ & 70.4 & 150 & & \\
\hline $8 \mathrm{H}-4,5-0.05$ & 70.55 & 230 & & \\
\hline $8 \mathrm{H}-4,15-0.15$ & 70.65 & 230 & & \\
\hline $8 \mathrm{H}-4,25-0.25$ & 70.75 & 205 & & \\
\hline $8 \mathrm{H}-4,35-0.35$ & 70.85 & 190 & & \\
\hline $8 \mathrm{H}-4,45-0.45$ & 70.95 & 210 & & \\
\hline $8 \mathrm{H}-4,55-0.55$ & 71.05 & 205 & & \\
\hline $8 \mathrm{H}-4,65-0.65$ & 71.15 & 185 & & \\
\hline $8 \mathrm{H}-4,75-0.75$ & 71.25 & 220 & + & 35 \\
\hline $8 \mathrm{H}-4,85-0.85$ & 71.35 & 385 & + & 25 \\
\hline $8 \mathrm{H}-4,95-0.95$ & 71.45 & 340 & + & 15 \\
\hline $8 \mathrm{H}-4,105-1.05$ & 71.55 & 285 & + & 5 \\
\hline $8 \mathrm{H}-4,110-1.1$ & 71.6 & 210 & + & 0 \\
\hline \multirow[t]{3}{*}{$8 \mathrm{H}-4,115-1.15$} & 71.65 & 285 & + & 5 \\
\hline & & & $\Sigma$ & 3335 \\
\hline & & & $\Sigma / 72$ & 46.32 \\
\hline
\end{tabular}

Note: Location of sapropels and the distance to the closed fault above or below are given in pocket penetrometer shear strength results section.

covered from the Cyprus slope (Site 968) had enhanced organic carbon values, which can be explained by rapid turbiditic deposition and burial as abundant turbidites also characterize this succession. Sapropel layers, which were found at all five sites, had significantly higher organic carbon contents that generally varied between $2 \%$ and $12 \%$. Occasionally, up to $24.6 \% \mathrm{C}_{\text {org }}$ was found (e.g., Pisano Plateau area, Site 964; Emeis, Robertson, Richter, et al., 1996). The sapropels in closest vertical proximity to the sapropel sample for shear box tests have carbonate contents of $6.8 \%$ (above) and $21.9 \%$ (below; Emeis, Robertson, Richter, et al., 1996).

Rock-Eval pyrolysis, however, obtained hydrogen indices that indicated that none of the sapropels contained entirely unaltered, primary marine organic matter. Furthermore, high $\mathrm{C}_{\text {org }} / \mathrm{N}$ ratios, varying from 10 up to 47 (maximum values also occurred at Site 964), were found at all five sites. It is suggested that diagenetic alteration must have affected removal of labile nitrogen-bearing organic compounds (amino acids) from the organic matter of the more deeply buried nannofossil oozes or sapropels. Possible explanations for the above findings include biological activity (bioturbation), microbial sulfate reducers (suggested by a high content of reduced sulfur), interaction with mineral-rich fluids, and/or diffusion of molecular oxygen from the sediment-water interface into sapropel beds (suggested by "burntdown" sapropels; Emeis, Robertson, Richter, et al., 1996).

\section{DISCUSSION Fault Abundance}

The comparison of fault abundance and variation of shear strength data suggest a possible correspondence between the position of faults and sapropels (i.e., elevated organic-carbon contents). About $95 \%$ of the total number of faults observed in the ten holes studied occurred in the sapropel-bearing intervals (see Fig. 3; Table 4). For the intervals of layered sediments chosen, the distance between a sapropel layer (including the "burnt-down" or oxidized sapropels; Emeis, Robertson, Richter, et al., 1996) and the closest fault was, on average, less than half a meter and never exceeded $4 \mathrm{~m}$ (Table 2 ). In intervals of the same cores that are sapropel-free (e.g., the light-gray nannofossil clays and oozes that typically underlie the sapropel bearing sediments), faults are absent or are found only rarely (Emeis, Robertson, Richter, et al., 1996). When faults are observed in the nannofossil ooze (sample core photographs $160-967 \mathrm{~B}-10 \mathrm{H}$ through $14 \mathrm{H}$ as examples; Emeis, Robertson, Richter, et al., 1996), their average halfdistance is about $1.5 \mathrm{~m}$ (Table 1). In the sapropel bearing intervals, normal faults often intersect sapropels (Table 2, last column, value = $0)$. Taking into account the small diameter of the core recovered and the generally steep angle of dip of the faults $\left(55-80^{\circ}\right.$; Kopf and Flecker, 1996), the extrapolated trace of a fault plane that does not cross-cut the sapropel on the core face is still likely to intersect a sapropel bed somewhere in the vicinity of the drill hole. Therefore, given that faulting is triggered by rheological contrasts, the majority of the penetrative faults in the sapropel bearing muds were initiated adjacent to sapropel beds.

Looking statistically at the shear strength results collected on a layer-by-layer basis adjacent to normal fault planes (vane and pocket penetrometer), three out of four measurements beneath the fault showed either lower or the same strength values as the layers above the fault (Table 5). Often, sapropel layers with their higher strength underlie the faults, while the weaker nannofossil oozes are faulted. Interestingly, similar patterns concerning sediment type and fault abundance were observed in successions recovered from the Japan Sea (Tamaki, Pisciotto, Allen, et al., 1990; Ingle Jr., Suyehiro, von Breymann et al., 1990). Moreover, during Leg 160 an almost identical series of normal faults with approximately the same distance to each other and the nearest sapropel was found by multiple coring in locations that were tens of meters apart from each other (see core photographs 160-964A-6H, 160-964B-6H, 160-964C-6H, and 160964D-6H, combined with correlation chart, "Site 964" chapter, Fig. 7, in Emeis, Robertson, Richter, et al., 1996). One possible explanation for this observation is that the region is permeated by a systematic set of conjugate normal faults, and that penetration of this meshwork, in whatever location, will produce a similar pattern of fault spacing. However, we believe that the distribution of normal faults here is simply a function of the force applied by the APC drilling device hitting a certain rigid layer at a certain depth. This problem has been considered earlier (e.g., Kopf, 1997), and the almost systematic pattern identified reflects the variation in sediment shear strength as a function of anisotropy of the deposits (Casagrande and Carrillo, 1953).

The strength measurements clearly illustrate why fault formation is found preferentially adjacent to rigid layers (Fig. 2A, B; Table 2) since they provide clear evidence for large strength contrasts. Unfortunately, no comparable closely spaced shear strength data are available from other ODP legs. Undrained shear strengths determined on interbedded successions of nannofossil ooze, silty clay, calcareous mud, and organic-rich, dark beds from ODP Leg 128 (Ingle, Suyehiro, von Breymann, et al., 1990) in the Japan Sea, however, range in the same order of magnitude as the data from Leg 160. Starting from values near zero at the sediment-water interface, values up to 30-90 $\mathrm{kPa}$ were usually reached at depths between 50 and $150 \mathrm{mbsf}$. The high values within these data were generally dark layers with $\mathrm{C}_{\text {org }}$ contents between $1.5 \%$ and $5.5 \%$. Both constant and linearly increasing trends with depth were found, which are in accordance with the majority of the data obtained during Leg 160 (see above and Table 2). On the other hand, equivalents of the high shear strength values of sapropel layers (up to $>200 \mathrm{kPa}$ ) were generally absent, although similar deposits were found (though not termed sapropels; Ingle, Suyehiro, von Breymann, et al., 1990).

Other studies on sub-surface sediments enriched in organic matter were carried out on samples from the eastern Japan Sea (Lee et al., 1993). Here, independent of the absolute shear strength values obtained from the gravity cores, a positive correlation between the amount of organic matter and vane shear strength was found at dif- 
A Pocket penetrometer:

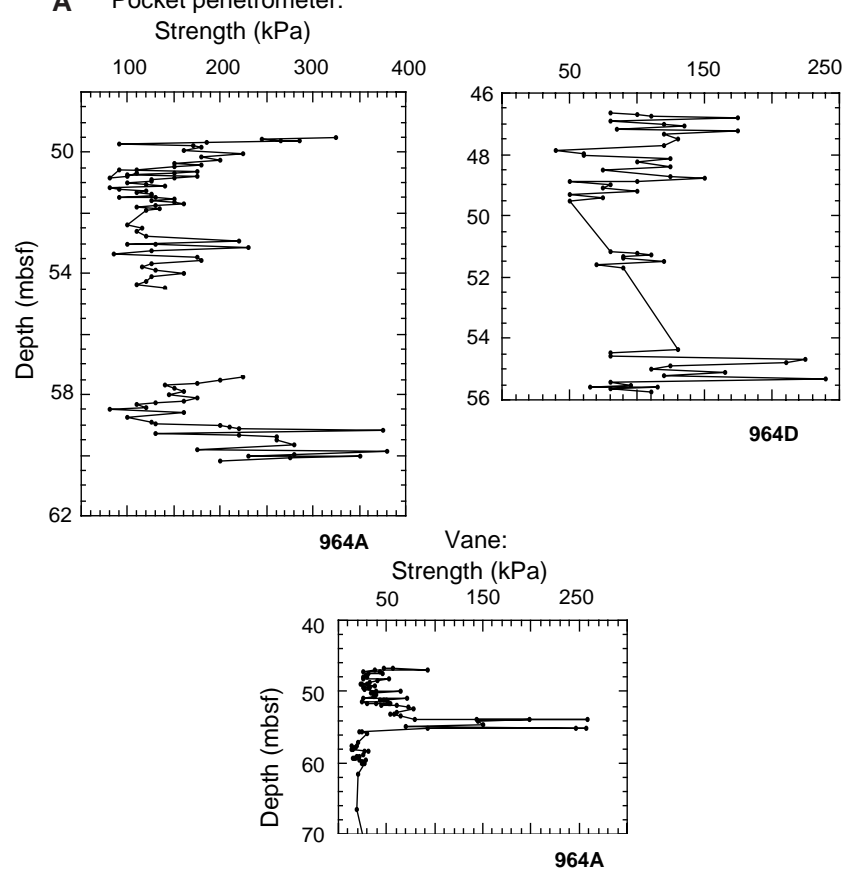

D Pocket penetrometer:
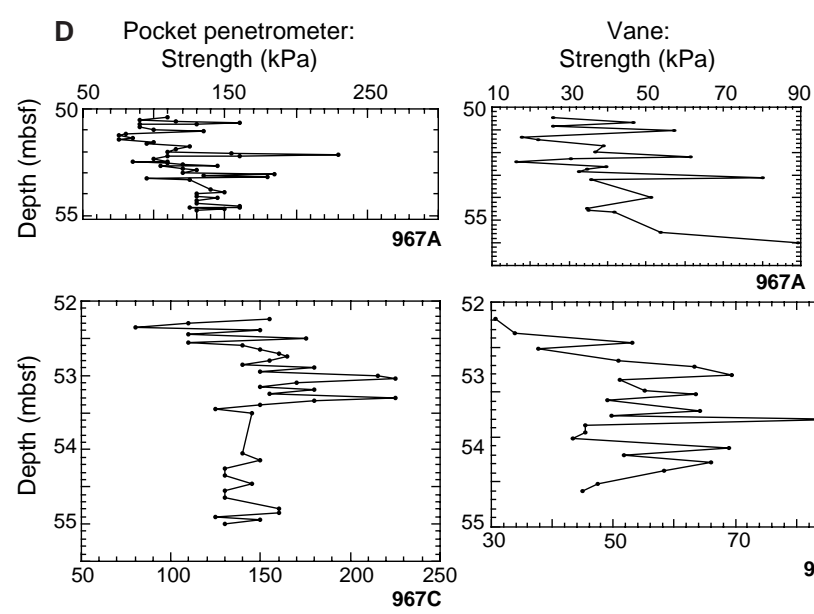

B Pocket penetrometer: Strength $(\mathrm{kPa})$
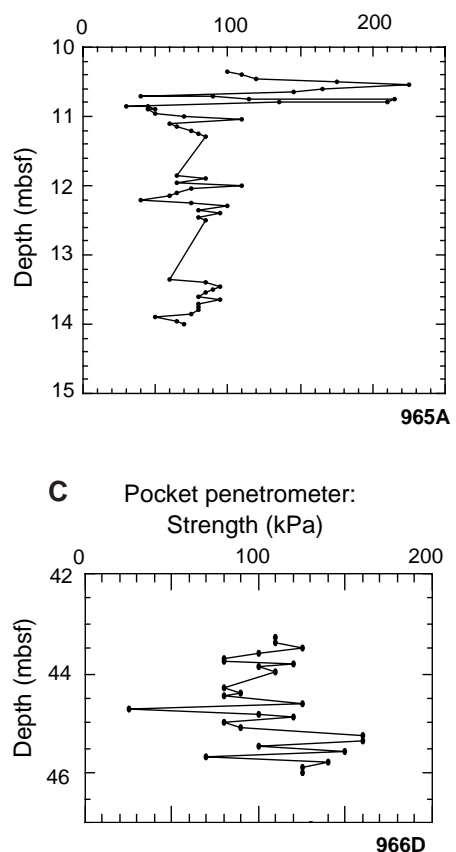

E Pocket penetrometer: Strength $(\mathrm{kPa})$

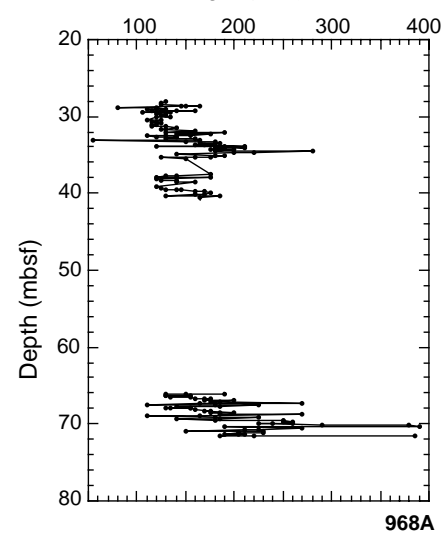

Vane: Strength $(\mathrm{kPa})$
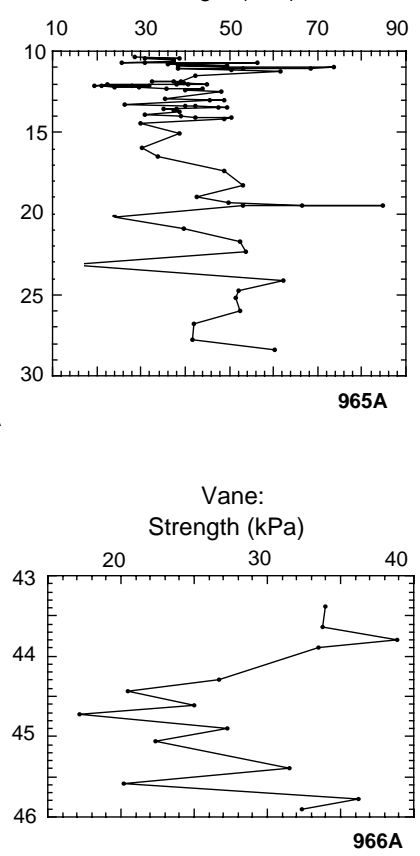

966A

Vane: Strength $(\mathrm{kPa})$

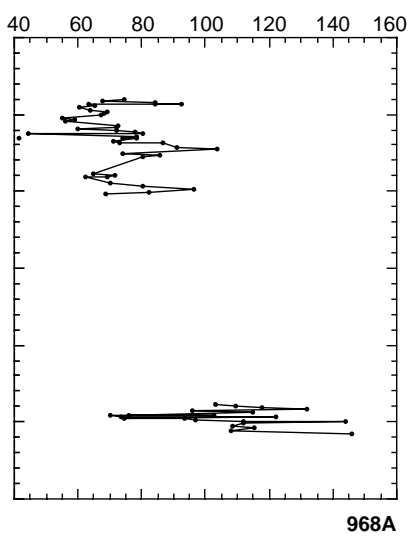

Figure 5. Summary figures of vane and pocket penetrometer shear strength measurements on a high-resolution basis. A. Holes 964A and 964D. B. Hole 965A. C. Holes 966A and 966D. D. Holes 967A and 967C. and E. Hole 968A. For full list of data see Table 1. Note that all diagrams vary in scale.

Table 3. Results of particle-size analysis and drained shear strength tests on remolded samples.

\begin{tabular}{|c|c|c|c|c|c|c|c|c|c|c|c|}
\hline \multirow[b]{2}{*}{ Sample } & \multirow[b]{2}{*}{$\begin{array}{l}\text { Depth } \\
\text { (mbsf) }\end{array}$} & \multicolumn{4}{|c|}{ Shear box tests } & \multicolumn{6}{|c|}{ Grain-size analyses } \\
\hline & & $\begin{array}{c}\text { Peak strength } \\
(\mathrm{kPa})\end{array}$ & $\begin{array}{l}\phi \mathrm{p} \\
\left({ }^{\circ}\right)\end{array}$ & $\begin{array}{l}\text { Residual strength } \\
\qquad(\mathrm{kPa})\end{array}$ & $\begin{array}{l}\phi \mathrm{r} \\
\left({ }^{\circ}\right)\end{array}$ & $\begin{array}{l}\text { Clay } \\
(\%)\end{array}$ & $\begin{array}{l}\text { Silt } \\
(\%)\end{array}$ & $\begin{array}{l}\text { Sand } \\
(\%)\end{array}$ & $\begin{array}{l}\text { Mean } \\
(\mu \mathrm{m})\end{array}$ & Skewness & $\begin{array}{l}\text { Standard } \\
\text { deviation }\end{array}$ \\
\hline $\begin{array}{l}\text { Nannofossil clay/ } \\
\text { nannofossil ooze }\end{array}$ & 25.35 & 621 & 30 & $<580$ & $<28.3$ & 23.35 & 76.65 & - & 30.19 & 2.21 & 49.42 \\
\hline Sapropel & 25.45 & 253 & 11.5 & $<226$ & $<10.3$ & 10.68 & 85.22 & 4.1 & 75.39 & 3.25 & 155.4 \\
\hline
\end{tabular}

Note: Both skewness and standard deviation of the grain-size data reflect a mismatch of the distribution to a Gaussian function. The standard deviation is known as the sorting of the deposits as outlined by previous workers (e.g., Camerlenghi et al., 1995). 
ferent locations. Similar results have been reported from the southern Central Pacific (Meadows et al., 1994), and the authors' conclusion was that particulate binding (possibly by extracellular, biogenic polymers at or near the sediment-water interface) caused higher strength. Even on land, the strength of soil has been found to increase with increasing organic content (Davies, 1985).

\section{Variability of the Strength as a Result of the Methods}

The vane device and pocket penetrometer determine undrained shear strength of sediment with its original fabric, while the drained geotechnical tests are carried out on remolded sediment. In the first case, the original cohesion and friction mobilized within the sediment with only nominal stress on the slip plane is measured, while the drained shear box test measures the strength per se (see discussion in Atkinson, 1993).

The strength values measured with the vane apparatus and pocket penetrometer were consistently higher for the sapropel beds than for the nannofossil oozes. However, the shear box values indicate that the sapropel has a lower drained shear strength than the nannofossil ooze sample. On one hand, this can be related to the different contents of (swelling) clay and organic matter in the samples discussion on composition below), because both can affect cohesion between parti-

\section{Concentration (\%wt)}

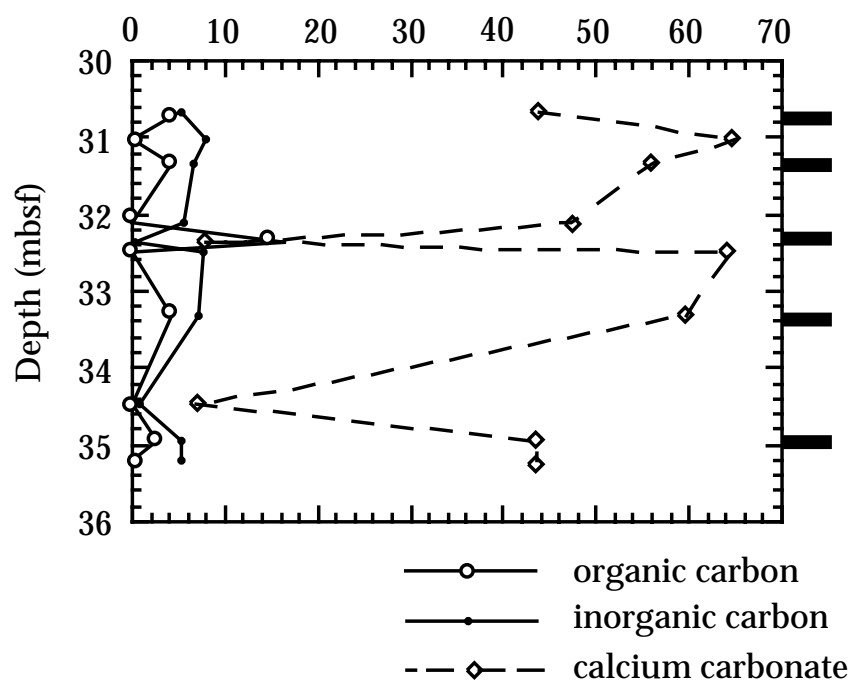

Figure 6. Total organic carbon, inorganic carbon, and $\mathrm{CaCO}_{3}$ contents over Sections 160-964A-4H-1 through 6, taken from shipboard organic geochemistry results of Leg 160, Sites 964-968 (Emeis, Robertson, Richter, et al., 1996). Locations of sapropels are marked with black bars in the right hand axis. See core photograph of the same interval above (Fig. 2A). cles, the plasticity index, and the frictional shear angle. On the other hand, the low value of drained strength of the remolded sapropel is consistent with the findings of previous studies where it has been shown that destruction of the original texture during remolding generally results in a loss of strength (e.g., Busch and Keller, 1981). Burland (1990) and Leroeuil and Vaughan (1990) have demonstrated that during remolding the higher order arrangements and bonding between particles are destroyed, and the sample develops a fabric, in terms of particle orientation, during the loading that precedes shear.
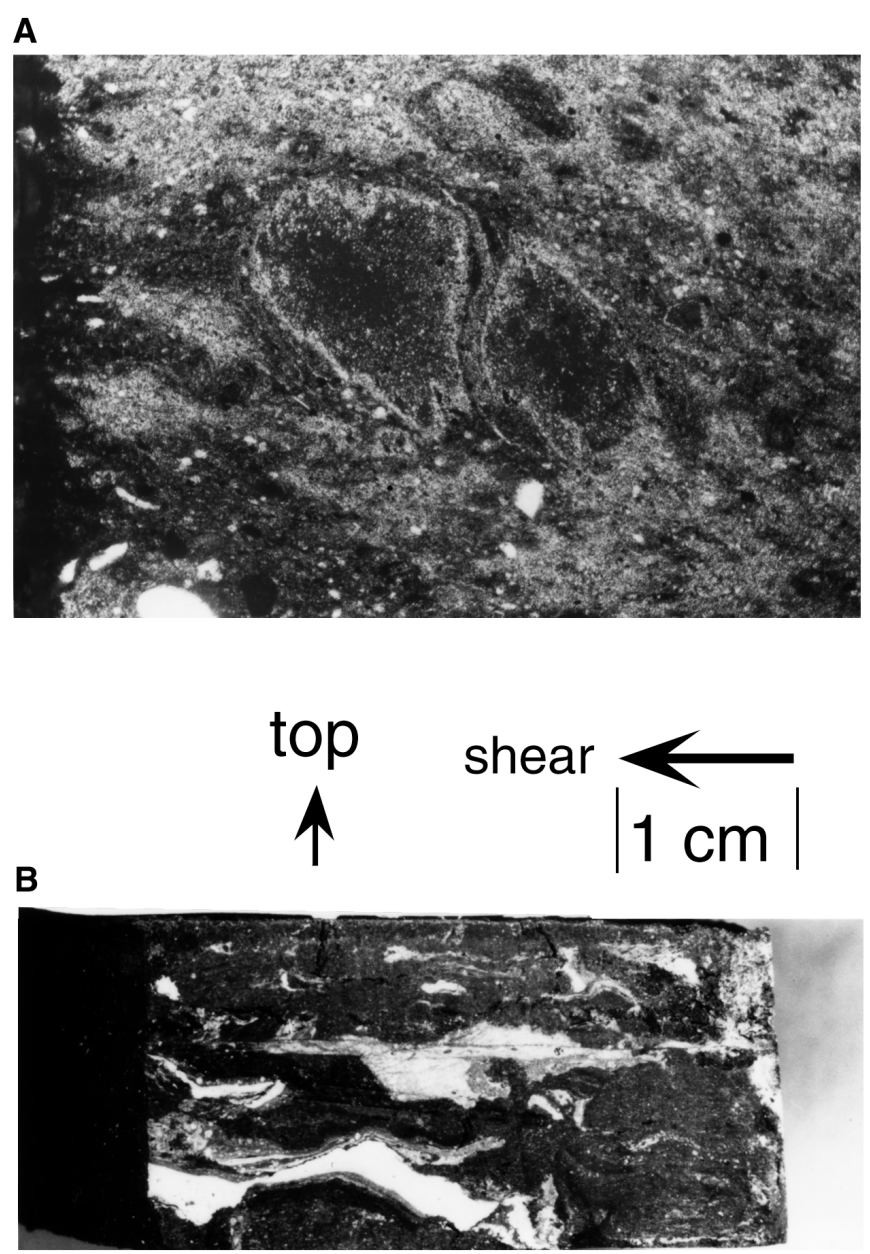

Figure 7. A. Thin-section photograph of the nannofossil ooze specimen, with darker material enriched pressure shadows of a foraminifer test. Long axis is $2.4 \mathrm{~mm}$. B. Photomicrograph of the sapropel sample after the geotechnical shear box test, with nannofossil ooze enriched along the shear planes.

Table 4. Abundance of faults and sapropels in APC-cored intervals as well as sapropel-bearing intervals.

\begin{tabular}{|c|c|c|c|c|c|c|}
\hline Hole & $\begin{array}{l}\text { Total length cored } \\
\text { with APC } \\
(\mathrm{m})\end{array}$ & $\begin{array}{l}\text { Number of } \\
\text { faults in } \\
\text { APC-interval }\end{array}$ & $\begin{array}{l}\text { Length of sapropel- } \\
\text { bearing succession } \\
\qquad(\mathrm{m})\end{array}$ & $\begin{array}{l}\text { Number of sapropels in } \\
\text { the sapropel-bearing } \\
\text { interval }\end{array}$ & $\begin{array}{l}\text { Number of faults in the } \\
\text { sapropel-bearing } \\
\text { interval }\end{array}$ & $\begin{array}{c}\text { Percentage of faults occurring in the } \\
\text { sapropel-bearing interval } \\
(\%)\end{array}$ \\
\hline $964 \mathrm{~A}$ & 101.8 & 47 & 82.8 & 35 & 47 & 100 \\
\hline $964 \mathrm{D}$ & 108.6 & 31 & 85 & 46 & 31 & 100 \\
\hline $965 \mathrm{~A}$ & 29.3 & 6 & 11 & 5 & 6 & 100 \\
\hline $966 \mathrm{~A}$ & 63.8 & 12 & 60 & 61 & 12 & 100 \\
\hline $966 \mathrm{C}$ & 95.1 & 26 & 82 & 86 & 26 & 100 \\
\hline $966 \mathrm{D}$ & 84.5 & 23 & 70 & 81 & 16 & 69.6 \\
\hline $967 \mathrm{~A}$ & 123.3 & 24 & 90.5 & 79 & 20 & 83.3 \\
\hline $967 \mathrm{~B}$ & 119.3 & 56 & 82.2 & 70 & 51 & 91.1 \\
\hline $967 \mathrm{C}$ & 114.4 & 16 & 76 & 70 & 16 & 100 \\
\hline $968 \mathrm{~A}$ & 85 & 82 & 85 & 56 & 82 & 100 \\
\hline
\end{tabular}

Note: The right column illustrates that the majority of the faults was found when sapropel beds were interbedded with the nanofossil ooze. 
Table 5. Summary of comparisons between results from the vane apparatus and the pocket penetrometer and shear strength values relative to the position of the normal fault plane, both for the vane apparatus and pocket penetrometer, on a site-by-site basis.

Summary

\begin{tabular}{lccc}
\hline Site & Vane $>\mathrm{pp}$ & Vane $=\mathrm{pp}$ & Vane $<\mathrm{pp}$ \\
\hline 964 & - & - & 10 \\
965 & - & $\overline{2}$ & 1 \\
966 & - & $\overline{1}$ & 3 \\
967 & $\overline{6}$ & 4 & 1 \\
968 & 6 & 7 & 24 \\
$\Sigma$ & $(16 \%)$ & $(19 \%)$ & $(65 \%)$ \\
& & &
\end{tabular}

Vane shear strength above and below normal fault planes

\begin{tabular}{lccc}
\hline Site & Above $>$ below Above $=$ below & Above $<$ below \\
\hline 964 & 3 & 2 & 5 \\
965 & 1 & 1 & $-\frac{2}{4}$ \\
966 & 1 & 1 & 4 \\
967 & - & 4 & 11 \\
968 & 9 & 8 & \\
$\Sigma$ & & & \\
\hline
\end{tabular}

Pocket penetrometer shear strength above and below normal fault planes

\begin{tabular}{llll}
\hline Site & \multicolumn{3}{c}{ Above $>$ below Above $=$ below Above $<$ below } \\
\hline 964 & 13 & - & 2 \\
965 & 1 & 1 & - \\
966 & 1 & - & 1 \\
967 & - & 5 & 3 \\
968 & 10 & 8 & 8 \\
$\Sigma$ & 25 & & \\
\hline
\end{tabular}

Vane apparatus and pocket penetrometer

$\begin{array}{cccc}\Sigma & 34 & 19 & 16 \\ & (50 \%) & (27 \%) & (23 \%)\end{array}$

Note: Two thirds of the measurements have similar or higher shear strength values above a fault plane which makes sense as failure develops from footwall to hanging wall. Only the 19 cores listed in Table 2 were taken into account. See text for discussion.

Some workers have shown that after remolding of fine-grained marine oozes, the strength decreased by more than an order of magnitude (Johnson et al., 1977).

A comparison between vane apparatus and pocket penetrometer has shown that measurements carried out with the latter device show systematically higher strengths, possibly as a result of a measurement bias (see "Physical Properties" section, "Explanatory Notes" chapter, Emeis, Robertson, Richter, et al., 1996). This assumption is supported by results from previous ODP legs where both techniques were used (e.g., Leg 146, Sites 889-892; Westbrook, Carson, Musgrave, et al., 1994). We conclude that the penetrometer is valuable for providing a quick and easy estimate of strength, and is useful where the vane device cannot be used. Although it is more reliable and refined than the penetrometer, previous studies have shown the limits of the vane apparatus. Specifically, the empirical factor $\mu$, which was introduced for strength estimates (Bjerrum, 1954), was revised and improved to $\mu_{v}$ (Morris and Williams, 1994). This factor is meant to compensate for disturbance and excess pore pressure when the device goes into the sediment, for excess pore pressure as a result of rotation, and for thixotropic effects caused by the rate of shearing.Matsui and Abe (1981) have shown the latter to be negligible, even though their data set of net pore pressures resulting from shearing is limited. Both the disturbance (e.g., Kimura and Saikoh, 1983) and excess pore pressure (Morris and Williams, 1993) were shown to be sufficient to considerably affect strength measurements. If the mineralogy of the sediment is known, however, a correlation between $\mu_{v}$, the plasticity index $I_{p}$, and the liquid limit $W_{L}$ exists (Morris and Williams, 1994). For this study, however, the Atterberg limits of the sediments were not determined on board the JOIDES Resolution during ODP Leg 160 , so that vane results could not be corrected afterward.

\section{Variability of the Strength as a Result of Sediment Fabric and Composition}

Structure may be particularly important where the textures formed in an anoxic environment such as that suggested for the formation of sapropels (e.g., Bradley, 1938; Williams et al., 1978). According to earlier work (e.g., van Olphen, 1963; Moon and Hurst, 1984), clay minerals are deposited with a preferred orientation in anoxic bottom water (i.e., poles on 001 parallel to the core axis), which leads to a higher sediment strength than that of sediments with honeycomb structures developed by flocculation out of oxic bottom water (Terzaghi, 1925). Layering on a millimeter to submillimeter basis was observed in many sapropels (Kemp et al., Chap. 27, this volume), and can be related to the suppression of biotic activity in an anoxic environment. Thus, the highest total organic carbon values were measured in the laminated sapropels (Emeis, Robertson, Richter, et al., 1996). The loss of strength caused by remolding of the sample may therefore have been partly compensated by higher organic carbon contents (sustaining the same shear strength despite higher water content; Rashid and Brown, 1975). Low sedimentation rates, as suggested from the positive relief of most of the drill locations selected (Emeis, Robertson, Richter, et al., 1996), may also contribute to the rigidity of these layers, as there are fewer particles affecting each other electrostatically during deposition and orientation and, as pore water, can escape more easily.

The mechanisms of how organic compounds, which are best preserved under anoxic conditions, influence the sediment strength is still the subject of controversy. Busch and Keller $(1981 ; 1982)$ related the apparent overconsolidation of $\mathrm{C}_{\mathrm{org}}$ rich deposits to particle bonding by organic debris. Kerogens, bitumens, or biofilms may increase sediment cohesiveness. By contrast, other workers found that decomposition and increasing humification of organic components, consolidation, and early diagenetic reactions were explanations for overconsolidation and stability of $\mathrm{C}_{\text {org }}$ rich sediment (Reimers, 1982; Lee et al., 1990). Organic-rich diatomaceous oozes were found to have higher shear strengths and consolidation stresses (Casagrande, 1936) than interbedded hemipelagic muds. The evaluation of the effect of organic matter on sediment shear strength using artificially prepared specimens also resulted in a positive relationship (e.g., Pusch, 1973; Rashid and Brown, 1975; Andersland et al., 1981), although possible influences of in situ overburden stress and age of the deposits (i.e., diagenetic effect on strength properties) could not be taken into account.

The angle of internal friction, $\phi$, as determined from the shear box tests, indicates that the sapropel's behavior is dominated by a clay mineral phase (e.g., Skempton, 1985). The clay, which was found still aggregated after dispersion in Calgon solution (see above), is likely to have settled slowly through the water column (see X-ray diffraction analyses on clay fraction in Robertson and Kopf, this volume). Both the clay and the nannofossil ooze of similar fine grain size must have been deposited during sapropel formation, and were then mobilized and localized along shear bands during shear box experiments (Fig. 7B). Arkin and Michaeli (1989) have shown that for artificial clay-carbonate mixtures, the angle of friction is mainly a function of the relationship between the components, grain size, and water content. For the initial water content of $\sim 50 \%$ (as determined for the sapropel after opening the sealed, cooled whole round sample), this relationship suggests a mixing ratio of carbonate to clay of 9:1 (arrow in Fig. 8), which would imply that there was a negligible amount of clay in the sample. The clay quantity increases if the carbonate frac- 


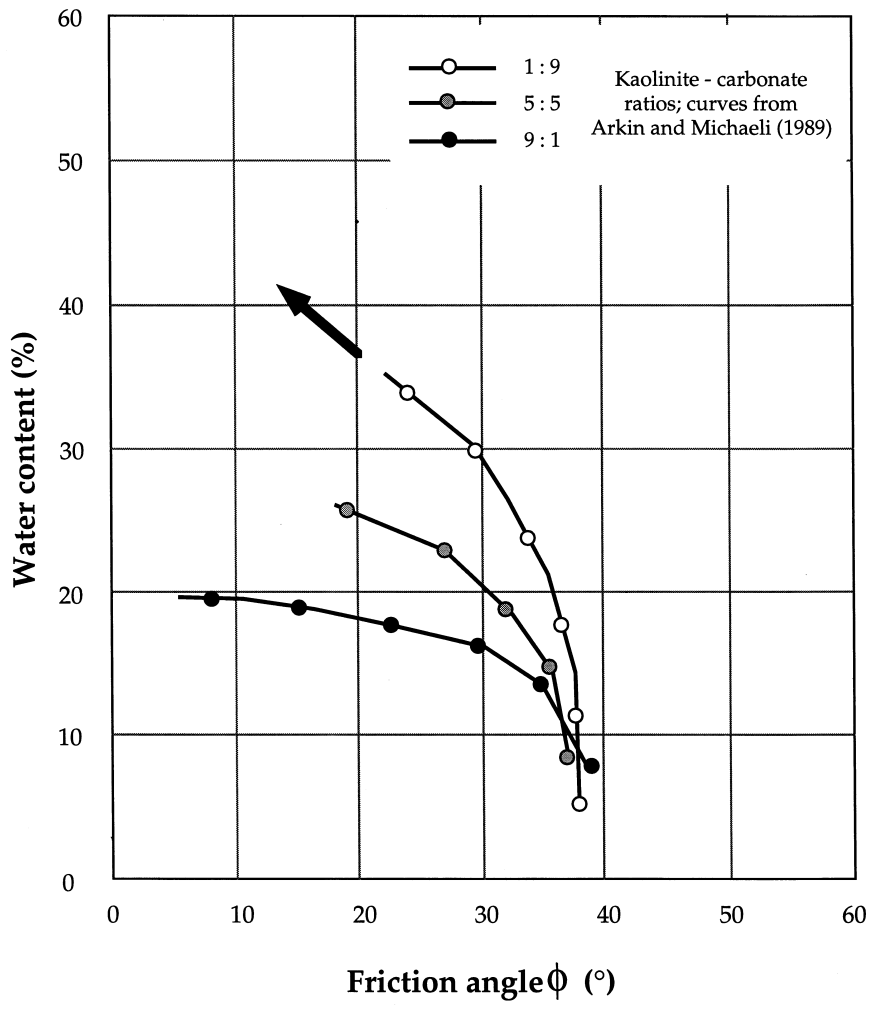

Figure 8. Curves from Arkin and Michaeli (1989), illustrating the variation of friction angle $\phi$ as a function of clay-carbonate ratio and water content. The arrow describes the extrapolated path towards a water content as high as approximately $50 \%$, the value determined for the sapropel specimen. The shaded area describes a field where finer grained carbonate compensates for (i.e., equals) the clay content (Arkin, 1986).

tion is very fine grained, as can be seen from the shaded area in Figure 8 (Arkin, 1986). If there is high contact area between particles (i.e., cohesion), as it is the case for the sapropel specimen studied, where finer particles are agglutinated, the curve is shifted towards a moderate clay-carbonate ratio (consistent with smear slide observations). Note, however, that friction angles determined from direct shear box tests are usually lower than those determined by triaxial tests (Atkinson et al., 1991).

\section{CONCLUSIONS}

From the results of this study and their discussion in the context of previous work we conclude that:

1. Shear strength measurements on nannofossil oozes and sapropels reveal differences, which seem to be independent of the three methods applied.

2. Higher undrained shear resistances on undisturbed sapropel beds were obtained using the vane apparatus and pocket penetrometer than on undisturbed nannofossil ooze. The drained strength value from shear box tests on a remolded sapropel was significantly lower than that for nannofossil ooze, probably because cohesion is less after the original fabric is destroyed.

3. Particle bonding and/or early post-sedimentary alteration of the organic matter are thought to be the most likely explana- tions for the rigidity of the sapropel beds. Also, in situ clay fabrics caused by deposition from an anoxic water column may have played a role in increasing rigidity, being mirrored by very high total organic carbon content.

4. The mobilization of a finer grained component during shear tests led to accumulation along shear zones and surfaces. In the sapropel it triggered separation of the organic-rich, black material and the admixed nannofossil ooze, which we have inferred to have been deposited as background sediment during sapropel accumulation.

5. A correlation between the organic carbon content and the undrained shear strength of sediments with their original fabric has been identified, and supports the conclusion of the majority of previous workers that there is a positive relationship between higher $\mathrm{C}_{\text {org }}$ content and higher shear resistance (e.g., Busch and Keller, 1981, 1982; Reimers, 1982; Davies, 1985; Meadows et al., 1994).

6. Faulting generally takes place within or adjacent to sapropel beds. Fault abundance can be positively correlated with the abundance of sapropels; intervals free of sapropels show minor or no faulting.

\section{ACKNOWLEDGMENTS}

Warner Brückmann and an anonymous reviewer are thanked for their critical comments and detailed suggestions. We would like to thank shipboard physical properties specialist Yossi Mart for carrying out additional vane shear experiments. Alastair Lumsden is acknowledged for use of the laboratory and geotechnical equipment at the University of Leeds. Support for this study was provided by DFG and NERC grants.

\section{REFERENCES}

Andersland, O.B., Khattak, A.S., and Al-Khafaji, A.W.N., 1981. Effect of organic material on soil shear strength. In Yong, R.N., and Townsend, R.C. (Eds.), Laboratory Shear Testing of Soil. Am. Soc. Testing and Materials, STP 740: 226-242.

Arkin, Y., 1986. Geotechnical factors influencing marl slopes in Israel. Isr. Geol. Surv., Jerusalem, 27/86.

Arkin, Y., and Michaeli, L., 1989. Strength and consistency of artificial claycarbonate mixtures: simulation of natural sediments. Eng. Geol., 26:201213.

American Safety Testing and Materials, 1987. Standard test method for laboratory miniature vane shear test for saturated fine-grained clayey soil. In Annual Book of ASTM Standards (Vol. 04.08): Philadelphia (Am. Soc. Testing and Mater.), D4648.

Atkinson, J., 1993. An Introduction to the Mechanics of Soils and Foundations: New York (McGraw-Hill).

Atkinson, J.H., Lau, W.H.W., and Powell, J.J.M., 1991. Measurement of soil strength in simple shear tests. Can. Geotech. J., 28:255-262.

Bjerrum, L., 1954. Geotechnical properties of Norwegian marine clays. Geotechnique, 4:49-69.

Borradaile, G.J., 1981. Particulate flow and the formation of cleavage. Tectonophysics, 72:305-321.

Boyce, R.E., 1976. Definitions and laboratory techniques of compressional sound velocity parameters and wet-water content, wet-bulk density, and porosity parameters by gravimetric and gamma-ray attenuation techniques. In Schlanger, S.O., Jackson, E.D., et al., Init. Repts. DSDP, 33: Washington (U.S. Govt. Printing Office), 931-958.

Bradley, W.H., 1938. Mediterranean sediments and Pleistocene sea levels. Science, 88:376-379.

British Standards (BS) 1377, 1991. Methods of Test for Soils for Civil Engineering Purposes: London (British Standards Institution).

Burland, J.B., 1990. On the compressibility and shear strength of natural clays. Geotechnique, 40:329-378. 
Busch, W.H., and Keller, G.H., 1981. The physical properties of Peru-Chile continental margin sediments: the influence of coastal upwelling on sediment properties. J. Sediment. Petrol., 51:705-719.

1982. Consolidation characteristics of sediments from the PeruChile Continental Margin and implications for past sediment instability. Mar. Geol., 45:17-39.

Camerlenghi, A., Lucchi, R.G., and Rothwell, R.G., 1995. Grain-size analysis and distribution in Cascadia Margin sediments, northeastern Pacific. In Carson, B., Westbrook, G.K., Musgrave, R.J., and Suess, E. (Eds.), Proc. ODP, Sci. Results, 146 (Pt 1): College Station, TX (Ocean Drilling Program), 3-31.

Casagrande, A., 1936. The determination of preconsolidation load and its practical significance. In Casagrande, A., Rutledge, P.C., and Watson, J.D. (Eds.), Proc. 1st Int. Conf. Soil Mech. Found. Eng., Am. Soc. Civ. Eng., 3:60-64.

Casagrande, A., and Carrillo, N., 1953. Shear failure of anisotropic materials. Contrib. Soil Mech. Boston Soc. Civil Engrs., 122-135.

Davies, P., 1985. Influence of organic matter content, moisture status and time after reworking on soil shear strength. J. Soil Sci., 36:299-306.

Demars, K.R., and Chaney, R.C. (Eds.), 1992. Geotechnical properties, behaviour and performance of calcareous soils. Am. Soc. for Testing and Mater., ASTM STP 777.

Emeis, K.-C., Robertson, A.H.F., Richter, C., et al., 1996. Proc. ODP, Init. Repts., 160: College Station, TX (Ocean Drilling Program).

Hubbert, M.K., 1951. Mechanical basis for certain familiar geologic structures. Geol. Soc. Am. Bull., 62:355-372.

Ingle, J.C., Jr., Suyehiro, K., von Breymann, M.T., et al., 1990. Proc. ODP, Init. Repts., 128: College Station, TX (Ocean Drilling Program).

Jaeger, J.C., and Cook, N.G.W., 1969. Fundamentals of Rock Mechanics: London (Methuen \& Co.).

Johnson, T.C., Hamilton, E.L., and Berger, W.H., 1977. Physical properties of calcareous ooze: control by dissolution at depth. Mar. Geol., 24:259277.

Jones, M.E., and Addis, M.A., 1986. The application of stress path and critical state analysis to sediment deformation. J. Struct. Geol., 8:575-580.

Kimura, T., and Saikoh, K., 1983. Effect of disturbance due to insertion on vane shear strength of normally consolidated cohesive soils. Soils Found., 23:113-124.

Knipe, R.J., 1986. Deformation mechanism paths for sediments undergoing lithification. Mem.-Geol. Soc. Am., 166:151-160.

Kopf, A., 1997. The origin, distribution, and related problems of interpreting normal faults in marine sediments recovered by Advanced Piston Coring (APC): a review of results from cruises of the Ocean Drilling Program (Legs 101-160). Sci. Drilling., 6:35-58.

Kopf, A., and Flecker, R., 1996. Problems associated with the interpretation of normal fault distributions in sediments recovered by the advanced hydraulic piston corer during Leg 160, Eastern Mediterranean. In Emeis, K.-C., Robertson, A.H.F., Richter, C., et al., Proc. ODP, Init. Repts., 160: College Station, TX (Ocean Drilling Program), 507-511.

Law, K.T., 1981. Effect of stress path geometry on soil brittleness. Geotechnique, 31:279-287.

Lee, H.J., 1985. State of the art: laboratory determination of the strength of marine soils. In Chaney, R.C., and Demars, K.R. (Eds.), Strength Testing of Marine Sediments: Laboratory and In-Situ Measurements: ASTM Spec. Tech. Publ., 883:181-250.

Lee, H.J., Chun, S.S., Yoon, S.H., and Kim, S.R., 1993. Slope stability and geotechnical properties of sediment of the southern margin of Ulleung Basin, East Sea (Sea of Japan). Mar. Geol., 110:31-45.

Lee, H.J., Kayen, R.E., and McArthur, W.G., 1990. Consolidation, triaxial shear-strength, and index-property characteristics of organic-rich sedi- ment from the Peru continental margin: results from Leg 112. In Suess, E., von Huene, R., et al., Proc. ODP, Sci. Results, 112: College Station, TX (Ocean Drilling Program), 639-651.

Lerouiel, S., and Vaughan, P.R., 1990. The general and congruent effects of structure in natural soils and rocks. Geotechnique, 40:467-488.

Maltman, A. (Ed.), 1994. The Geological Deformation of Sediments: London (Chapman \& Hall).

Matsui, T., and Abe, N., 1981. Shear mechanisms of vane tests in soft clays. Soils Found., 21:69-80.

Meadows, P.S., Reichelt, A.C., Meadows, A., and Waterworth, J.S., 1994. Microbial and meiofaunal abundance, redox potential, $\mathrm{pH}$ and shear strength profiles in deep sea Pacific sediments. J. Geol. Soc. London, 151:377-390.

Moon, C.F., and Hurst, C.W., 1984. Fabrics of muds and shales: an overview. In Stow, D.A.V., and Piper, D.J.W. (Eds.), Fine-Grained Sediments: Deep-Water Processes and Facies. Geol. Soc. Spec. Publ. London, 15:579-593.

Morris, P.H., and Williams, D.J., 1993. A new model of vane shear strength testing in soils. Geotechnique, 43:489-500.

, 1994. Effective stress vane shear strength correction factor correlations. Can. Geotech. J., 31:335-342.

Pusch, R., 1973. Influence of organic matter on the geotechnical properties of clays. Natl. Swed. Bldg. Res., 11.

Ramsay, J.G., and Huber, M.I., 1987. The Techniques of Modern Structural Geology (Vol. 2): Folds and Fractures: New York (Academic Press).

Rashid, M.A., and Brown, J.D., 1975. Influence of marine organic compounds on the engineering properties of a remolded sediment. Engr. Geol., 9:141-154.

Reimers, C.E., 1982. Organic matter in anoxic sediments off Central Peru: Relations of porosity, microbial decomposition and deformation properties. Mar. Geol., 46: 175-197.

Roscoe, K.H., 1953. An apparatus for the application of simple shear to soil samples. Proc. 3rd Int. Conf. Soil Mech. Found. Eng., Zürich, 1:86-191.

Skempton, J.A.W., 1985. Residual strength of clays in landslides, folded strata and the laboratory. Geotechnique, 35:3-18.

Tamaki, K., Pisciotto, K., Allan, J., et al., 1990. Proc. ODP, Init. Repts., 127 College Station, TX (Ocean Drilling Program).

Tucker, M.E., 1981. An Introduction to Sedimentary Petrology: Oxford (Blackwell).

van Olphen, H., 1963. An Introduction to Clay Colloid Chemistry: New York/London (Interscience Publ.)

Westbrook, G.K., Carson, B., Musgrave, R.J., et al., 1994. Proc. ODP, Init. Repts., 146 (Pt. 1): College Station, TX (Ocean Drilling Program).

Williams, D.F., Thunell, R.C., and Kennett, J.P., 1978. Periodic freshwater flooding and stagnation of the eastern Mediterranean Sea during the late Quaternary. Science, 201:252-254.

Wood, D.M., 1990. Soil Behaviour and Critical State Soil Mechanics: Cambridge (Cambridge Univ. Press).

Yassir, N., 1990. The undrained shear behavior of fine sediments. In Knipe, R.J., and Rutter, E.H. (Eds.), Deformation Mechanisms, Rheology and Tectonics. Geol. Soc. Spec. Publ. London, 54:399-404.

Date of initial receipt: 27 January 1997

Date of acceptance: 4 August 1997

Ms 160SR-050 